\title{
ON A STOCHASTIC WAVE EQUATION WITH UNILATERAL BOUNDARY CONDITIONS
}

\author{
JONG UHN KIM
}

\begin{abstract}
We prove the existence and uniqueness of solutions to the initial boundary value problem for a one-dimensional wave equation with unilateral boundary conditions and random noise. We also establish the existence of an invariant measure.
\end{abstract}

\section{$\S 0$. INTRODUCTION}

In this paper, we discuss a one-dimensional wave equation with unilateral boundary conditions and random noise. The problem is formulated as follows:

$$
\begin{gathered}
u_{t t}-u_{x x}+\alpha u_{t}=\frac{\partial \Phi}{\partial t}, \quad \text { for }(x, t) \in(0, L) \times(0, T), \\
u_{x}(0, t) \leq 0, \quad u(0, t) \geq 0, \quad u_{x}(0, t) u(0, t)=0, \quad \text { for } t \in(0, T), \\
u(L, t)=0, \quad \text { for } t \in(0, T), \\
u(x, 0)=u_{0}(x), \quad u_{t}(x, 0)=u_{1}(x), \quad \text { for } x \in(0, L) .
\end{gathered}
$$

This problem is associated with the longitudinal motion of a linear elastic bar against a rigid stationary obstacle. Here $u$ denotes the axial displacement, and the boundary condition (0.2) expresses the condition that the left end of the bar does not stick to the obstacle and the rigid obstacle can exert stress only if the bar is in contact with the obstacle. $\Phi$ is a continuous martingale which will be described in detail later, and $\alpha$ is a nonnegative constant. For the case when $\partial_{t} \Phi$ is replaced by a deterministic function and the damping term $\alpha u_{t}$ is replaced by a memory term, this initial-boundary value problem has been investigated in [9]. Related deterministic problems were studied in [3], [4], [7], [12], [13] and [15]. However, with a random noise, this is a completely new problem, which is not readily covered by the existing results. For general results on the stochastic evolution equations, the readers are referred to [1]. The nonlinear boundary condition (0.2) combined with a random noise poses a new challenge in stochastic analysis. Our goal is to prove the existence and uniqueness of solutions to (0.1) - (0.4), and to establish the existence of an invariant measure.

For a parabolic equation with unilateral constraint, the existence and uniqueness of solutions can be obtained through the form of a stochastic parabolic variational inequality; see [5], where the penalty method was used. We will also use the penalty

\footnotetext{
Received by the editors June 9, 2004 and, in revised form, July 17, 2005.

2000 Mathematics Subject Classification. Primary 35L65, 35R60, $60 \mathrm{H} 15$.

Key words and phrases. Unilateral boundary conditions, Brownian motion, existence of a solution, pathwise uniqueness, invariant measure, probability distribution.
} 
method which is meaningful from the physical viewpoint. Namely, we approximate the rigid obstacle by elastic obstacles with increasing rigidity and obtain a solution as a limit of the approximate solutions. So our general strategy is fairly standard. However, as the rigidity parameter increases to infinity, we do not have strong convergence of approximate solutions. We only have weak convergence over the sample space. This is in sharp contrast to the parabolic case in [5]. Weak convergence over the sample space is not sufficient to obtain pathwise solutions of our problem. This is the main reason why we will work directly with the above form $(0.1)-(0.4)$ even though the problem can be formulated as a stochastic hyperbolic variational inequality. For a deterministic nonlinear problem, we can derive strong convergence in a larger function class from weak convergence by means of compact imbedding of function spaces to handle nonlinearity. In the case of stochastic problems, weak convergence over the sample space cannot be translated into strong convergence. The nonlinearity is due to the boundary condition (0.2). In particular, weak convergence is not sufficient for a limit function to satisfy the last condition in (0.2). Fortunately, we have some partial strong convergence of the trace of approximate solutions as rigidity tends to infinity. This requires some unusual estimates, which are also essential for the proof of pathwise uniqueness. One of our goals is to address these technical issues. If the noise term is additive, i.e., $\Phi$ is independent of the unknown function $u$, then the technical procedure can be simpler. In this case, we have pathwise convergence of approximate solutions by splitting each approximate solution into two parts. Namely, one part takes care of the random noise, and the other part is a solution of essentially a deterministic problem. This will be shown in Section 5 below.

For asymptotic behavior of solutions to stochastic evolution equations, an invariant measure is an important object. If the probability law of the initial data is the same as an invariant measure, then the probability law of evolving solutions is invariant in time. This corresponds to stationary solutions of deterministic equations. There are well-known results on the existence of invariant measures for general semi-linear evolution equations: see [1] and [2]. But the existence of an invariant measure for $(0.1)-(0.3)$ is a completely new problem which is not covered by these well-known results. Recently, the author [10] obtained a new existence result on the invariant measures of a certain class of evolution equations. It turns out that the above problem (0.1) - (0.3) fits into this class. Our task is to show that all the required assumptions for the result in [10] are satisfied. For this, we need pathwise convergence of approximate solutions to justify necessary conditions for the result in [10]. Hence, we can handle only the case of an additive noise for the existence of an invariant measure.

Finally, we note that a stochastic version of the problem discussed in [12] and [13] is still an open question. When an obstacle is placed in the interior, the uniqueness of the solution is known only under an extra condition of energy conservation. It is not known how such a condition can be modified for the stochastic problem.

\section{$\S 1$. Notation And Statement of the main Results}

We will use the following notation:

$$
\partial_{t} u=u_{t}=\frac{\partial u}{\partial t}, \quad \partial_{x} u=u_{x}=\frac{\partial u}{\partial x},
$$


and

$$
h^{+}=\max (h, 0), \quad h^{-}=\max (-h, 0) .
$$

For a real number $s, H^{s}(0, L)$ denotes the usual Sobolev space of order $s$ over the interval $(0, L)$. If $h \in H^{1}(0, T)$, then $h(\cdot)^{-} \in H^{1}(0, T)$ and

$$
\frac{d}{d t}\left(h(\cdot)^{-}\right)=-\chi\{h(t)<0\} \frac{d}{d t} h(\cdot)
$$

where $\chi\{\cdots\}$ is the characteristic function of the set $\{\cdots\}$.

$\left(\Omega, \mathcal{F}, \mathcal{F}_{t}, P\right)$ is a given stochastic basis, where $P$ is a probability measure, $\mathcal{F}$ is a $\sigma$-algebra and $\left\{\mathcal{F}_{t}\right\}_{t \geq 0}$ is a right-continuous filtration on $(\Omega, \mathcal{F})$ such that $\mathcal{F}_{0}$ contains all $P$-negligible subsets. $\left\{B_{j}(t)\right\}_{j=1}^{\infty}$ is a sequence of mutually independent standard Brownian motions over $\left(\Omega, \mathcal{F}, \mathcal{F}_{t}, P\right) . E(\cdot)$ stands for expectation with respect to the probability measure $P$. In this paper, a stochastic integral is defined in the sense of Ito. When $\mathcal{O}$ is a topological space, $\mathcal{B}(\mathcal{O})$ denotes the Borel $\sigma$ algebra over $\mathcal{O}$. When $\mathcal{X}$ is a Banach space, an $\mathcal{X}$-valued function $f$ is said to be $\mathcal{F}$-measurable if $f^{-1}(G) \in \mathcal{F}$ for every $G \in \mathcal{B}(\mathcal{X})$. This coincides with strong measurability for Bochner integrals when the range of $f$ is separable. When $\mathcal{X}$ is a Banach space, $L^{p}(\Omega ; \mathcal{X}), 1 \leq p<\infty$, denotes the set of all $\mathcal{X}$-valued strongly measurable functions such that

$$
\int_{\Omega}\|f\|_{\mathcal{X}}^{p} d P<\infty
$$

An $\mathcal{X}$-valued stochastic process $Y(t)$ is said to be progressively measurable if $Y$ restricted to the interval $[0, t]$ is $\mathcal{B}([0, t]) \otimes \mathcal{F}_{t}$-measurable for each $t \geq 0$. If $\mathcal{X}$ is a separable Hilbert space, then $L^{\infty}(0, T ; \mathcal{X})$ is the dual of $L^{1}(0, T ; \mathcal{X})$. In this case, let $L_{*}^{2}\left(\Omega ; L^{\infty}(0, T ; \mathcal{X})\right)$ be the set of all $f$ such that $\langle f, q\rangle$ is $\mathcal{F}$-measurable for every $q \in L^{1}(0, T ; \mathcal{X})$, where $\langle\cdot, \cdot\rangle$ is the duality pairing between $L^{\infty}(0, T ; \mathcal{X})$ and $L^{1}(0, T ; \mathcal{X})$, and

$$
\int_{\Omega}\|f(\omega)\|_{L^{\infty}(0, T ; \mathcal{X})}^{2} d P<\infty
$$

Then, $L_{*}^{2}\left(\Omega ; L^{\infty}(0, T ; \mathcal{X})\right)$ is the dual of $L^{2}\left(\Omega ; L^{1}(0, T ; \mathcal{X})\right)$; see [14].

Throughout this paper, we suppose that

$$
\Phi=\Phi(u)(t)=\sum_{j=1}^{\infty} \int_{0}^{t}\left(\sigma_{j} u+f_{j}\right) d B_{j}
$$

where the $f_{j}$ 's are progressively measurable with respect to $\left\{\mathcal{F}_{t}\right\}$, and

$$
\sum_{j=1}^{\infty}\left\|f_{j}\right\|_{L^{2}\left(\Omega ; L^{2}\left(0, T ; L^{2}(0, L)\right)\right.}^{2}<\infty
$$

for each $T>0$. The $\sigma_{j}$ 's are deterministic functions such that

$$
\sum_{j=1}^{\infty}\left\|\sigma_{j}\right\|_{L^{\infty}((0, L) \times(0, T))}^{2}<\infty
$$

for each $T>0$. 
Lemma 1.1. Suppose that $\left\{v_{k}\right\}_{k=1}^{\infty}$ is a sequence of progressively measurable functions in $L^{2}\left(\Omega ; L^{2}\left(0, T ; L^{2}(0, L)\right)\right)$ such that as $k \rightarrow \infty$,

$$
\begin{aligned}
v_{k} & \rightarrow v \quad \text { weakly in } L^{2}\left(\Omega ; L^{2}\left(0, T ; L^{2}(0, L)\right)\right), \\
\partial_{t} v_{k} & \rightarrow \partial_{t} v \quad \text { weakly in } L^{2}\left(\Omega ; L^{2}\left(0, T ; L^{2}(0, L)\right)\right) .
\end{aligned}
$$

Then, as $k \rightarrow \infty$,

$$
\sum_{j=1}^{\infty} \int_{0}^{(\cdot)} \sigma_{j} v_{k} d B_{j} \rightarrow \sum_{j=1}^{\infty} \int_{0}^{(\cdot)} \sigma_{j} v d B_{j}
$$

weakly in $L^{2}\left(\Omega ; L^{2}\left(0, T ; L^{2}(0, L)\right)\right)$.

Proof. For any $\epsilon>0$, there is a positive integer $N$ such that

$$
\sum_{j=N}^{\infty} E\left(\int_{0}^{T}\left\|\sigma_{j} v_{k}\right\|_{L^{2}(0, L)}^{2} d t\right)<\epsilon
$$

for all $k \geq 1$. Hence, it is enough to show that as $k \rightarrow \infty$,

$$
\int_{0}^{(\cdot)} \sigma_{j} v_{k} d B_{j} \rightarrow \int_{0}^{(\cdot)} \sigma_{j} v d B_{j}
$$

weakly in $L^{2}\left(\Omega ; L^{2}\left(0, T ; L^{2}(0, L)\right)\right)$, for each $j$. Since $\partial_{t} v_{k} \in L^{2}\left(\Omega ; L^{2}(0, T\right.$; $\left.\left.L^{2}(0, L)\right)\right)$, we have

$$
d v_{k}=\left(\partial_{t} v_{k}\right) d t
$$

which, combined with the Ito calculus (see [8]), yields

$$
v_{k}(t) \int_{0}^{t} \sigma_{j}(s) d B_{j}(s)=\int_{0}^{t} v_{k}(s) \sigma_{j}(s) d B_{j}(s)+\int_{0}^{t}\left(\int_{0}^{s} \sigma_{j}(\eta) d B_{j}(\eta)\right) \partial_{s} v_{k}(s) d s
$$

for all $t \in[0, T]$, for almost all $\omega \in \Omega$. Since we have

$$
\int_{0}^{(\cdot)} \sigma_{j}(s) d B_{j}(s) \in L^{2}\left(\Omega ; C\left([0, T] ; L^{2}(0, L)\right)\right),
$$

it holds that for any $b \in L^{2}\left(0, T ; L^{\infty}(0, L)\right)$ and $G \in \mathcal{F}$,

$\int_{G} \int_{0}^{T}\left\langle b(t), v_{k}(t) \int_{0}^{t} \sigma_{j}(s) d B_{j}(s)\right\rangle d t d P \rightarrow \int_{G} \int_{0}^{T}\left\langle b(t), v(t) \int_{0}^{t} \sigma_{j}(s) d B_{j}(s)\right\rangle d t d P$ as $k \rightarrow \infty$. Here $\langle\cdot, \cdot\rangle$ denotes the duality pairing between $L^{1}(0, L)$ and $L^{\infty}(0, L)$.

By changing the order of integration, we see that

$$
\begin{aligned}
\int_{G} \int_{0}^{T}\left\langle b(t), \int_{0}^{t}\left(\int_{0}^{s} \sigma_{j}(\eta) d B_{j}(\eta)\right) \partial_{s} v_{k}(s) d s\right\rangle d t d P \\
\quad=\int_{G} \int_{0}^{T}\left\langle\int_{t}^{T} b(s) d s, \partial_{t} v_{k}(t) \int_{0}^{t} \sigma_{j}(s) d B_{j}(s)\right\rangle d t d P
\end{aligned}
$$

and hence,

$$
\begin{aligned}
\int_{G} \int_{0}^{T}\left\langle b(t), \int_{0}^{t}\left(\int_{0}^{s} \sigma_{j}(\eta) d B_{j}(\eta)\right) \partial_{s} v_{k}(s) d s\right\rangle d t d P \\
\quad \rightarrow \int_{G} \int_{0}^{T}\left\langle b(t), \int_{0}^{t}\left(\int_{0}^{s} \sigma_{j}(\eta) d B_{j}(\eta)\right) \partial_{s} v(s) d s\right\rangle d t d P
\end{aligned}
$$


as $k \rightarrow \infty$. Therefore, it follows from (1.5) that

$$
\begin{aligned}
\int_{G} \int_{0}^{T}\left\langle b(t), \int_{0}^{t} v_{k}(s) \sigma_{j}(s) d B_{j}(s)\right\rangle d t d P \\
\quad \rightarrow \int_{G} \int_{0}^{T}\left\langle b(t), \int_{0}^{t} v(s) \sigma_{j}(s) d B_{j}(s)\right\rangle d t d P \quad \text { as } k \rightarrow \infty
\end{aligned}
$$

for every $b \in L^{2}\left(0, T ; L^{\infty}(0, L)\right)$ and $G \in \mathcal{F}$. We note that

$$
\int_{0}^{(\cdot)} \sigma_{j} v_{k} d B_{j} \quad \text { is bounded in } L^{2}\left(\Omega ; C\left([0, T] ; L^{2}(0, L)\right)\right) \text { uniformly in } k,
$$

and that each function in $L^{2}\left(\Omega ; L^{2}\left(0, T ; L^{2}(0, L)\right)\right)$ can be approximated by functions of the form $\sum_{i=1}^{N} b_{i} \chi\left\{G_{i}\right\}$, where $1 \leq N<\infty, b_{i} \in L^{2}\left(0, T ; L^{\infty}(0, L)\right)$ and $G_{i} \in \mathcal{F}$. Consequently, (1.4) follows from (1.6).

Next we set

$$
\Psi(t)=\sum_{j=1}^{\infty} \int_{0}^{t} f_{j}(s) d B_{j}(s)
$$

and recall the following known fact.

Lemma 1.2. For any $\psi \in C_{0}^{\infty}((0, T))$, it holds that

$$
\int_{0}^{T} \psi_{t}(t) \Psi(t) d t=-\sum_{j=1}^{\infty} \int_{0}^{T} \psi(t) f_{j}(t) d B_{j}(t)
$$

for almost all $\omega \in \Omega$.

Proof. Choose any $\psi \in C_{0}^{\infty}((0, T))$, and set

$$
\Psi_{m}(t)=\sum_{j=1}^{m} \int_{0}^{t} f_{j}(s) d B_{j}(s) .
$$

Let us partition the interval $[0, T]$ as

$$
0=t_{0}<t_{1}<\cdots<t_{N}=T, \quad t_{k}-t_{k-1}=\delta_{N}=T / N, \quad k=1, \cdots, N,
$$

and define

$$
\psi_{N}(t)=\psi\left(t_{j}\right), \quad \text { for } t_{j-1}<t \leq t_{j}, 1 \leq j \leq N .
$$

Since $\Psi_{m} \in C\left([0, T] ; L^{2}(0, L)\right)$, for almost all $\omega$, and

$$
\frac{\psi\left(t+\delta_{N}\right)-\psi(t)}{\delta_{N}} \rightarrow \psi_{t}(t) \quad \text { uniformly in } t \in[0, T] \text { as } N \rightarrow \infty,
$$

we have

$$
\begin{aligned}
& \int_{0}^{T} \psi_{t}(t) \Psi_{m}(t) d t=\lim _{N \rightarrow \infty} \sum_{k=0}^{N-1} \frac{\psi\left(t_{k+1}\right)-\psi\left(t_{k}\right)}{\delta_{N}} \Psi_{m}\left(t_{k}\right) \delta_{N} \\
& =-\lim _{N \rightarrow \infty} \sum_{k=1}^{N-1} \psi\left(t_{k}\right)\left(\Psi_{m}\left(t_{k}\right)-\Psi_{m}\left(t_{k-1}\right)\right) \\
& =-\lim _{N \rightarrow \infty} \sum_{j=1}^{m} \int_{0}^{T} \psi_{N}(t) f_{j}(t) d B_{j}(t),
\end{aligned}
$$


for almost all $\omega$. In the meantime, as $N \rightarrow \infty$,

$$
\sum_{j=1}^{m} \int_{0}^{T} \psi_{N}(t) f_{j}(t) d B_{j}(t) \rightarrow \sum_{j=1}^{m} \int_{0}^{T} \psi(t) f_{j}(t) d B_{j}(t)
$$

in $L^{2}\left(\Omega ; L^{2}(0, L)\right)$. Hence,

$$
\int_{0}^{T} \psi_{t}(t) \Psi_{m}(t) d t=-\sum_{j=1}^{m} \int_{0}^{T} \psi(t) f_{j}(t) d B_{j}(t)
$$

for almost all $\omega \in \Omega$.

By passing $m \rightarrow \infty$, we arrive at (1.7).

Definition 1.3. A progressively measurable function $u \in L^{2}\left(\Omega ; L^{2}\left(0, T ; H^{1}(0, L)\right)\right)$ is said to be a solution of $(0.1)-(0.4)$ if for almost all $\omega \in \Omega$, it satisfies $(0.1)$ in the sense of distributions over $(0, L) \times(0, T),(0.2)-(0.3)$ for almost all $t \in[0, T]$, and (0.4) for almost all $x \in[0, L]$.

Our main results are as follows.

Theorem 1.4. Let $\alpha \geq 0$, and suppose that $u_{0}$ and $u_{1}$ are $\mathcal{F}_{0}$-measurable such that $u_{1} \in L^{2}\left(\Omega ; L^{2}(0, L)\right)$ and $u_{0} \in L^{2}\left(\Omega ; H^{1}(0, L)\right)$ with $u_{0}(0) \geq 0$ and $u_{0}(L)=0$, for almost all $\omega \in \Omega$. For any $T>0$, there is a pathwise unique solution $u$ of (0.1) (0.4) such that $u$ is progressively measurable, and

$$
u \in L^{2}\left(\Omega ; C\left([0, T] ; H^{1}(0, L)\right)\right), \quad u_{t} \in L^{2}\left(\Omega ; C\left([0, T]: L^{2}(0, L)\right)\right) .
$$

Theorem 1.5. Let $\alpha>0$, and assume that $\sigma_{j} \equiv 0, j \geq 1$, and that the $f_{j}$ 's are independent of time. Then, there is an invariant measure of $(0.1)-(0.3)$ over $H^{1}(0, L) \times L^{2}(0, L)$.

\section{§2. Deterministic equation}

In this section, we will present basic facts on the deterministic equation which will be used for the proof of Theorem 1.4.

Lemma 2.1. Suppose that

$$
v \in L^{2}\left(0, T ; H^{1}(0, L)\right), \quad v_{t} \in L^{2}\left(0, T ; L^{2}(0, L)\right)
$$

and that $v$ satisfies

$$
v_{t t}-v_{x x}+\alpha v_{t}=0 \quad \text { for }(x, t) \in(0, L) \times(0, T)
$$

and

$$
v(x, 0)=0, \quad v_{t}(x, 0)=0 \quad \text { for } x \in(0, L) .
$$

Then, it holds that for each $0<\epsilon<T$,

$$
v \in C\left([0, T-\epsilon] ; H^{1}(0, L)\right), \quad v_{t} \in C\left([0, T-\epsilon] ; L^{2}(0, L)\right)
$$

and

$$
\begin{array}{ll}
v_{x}(0, \cdot), & v_{x}(L, \cdot) \in L^{2}(0, T-\epsilon), \\
v_{t}(0, \cdot), & v_{t}(L, \cdot) \in L^{2}(0, T-\epsilon) .
\end{array}
$$

This is a special case of Lemma 1.6 of [9]. Here (2.2) and (2.3) are satisfied in the sense of distributions over $(0, L) \times(0, T)$ and $(0, L)$, respectively. 
Lemma 2.2. For given $q \in L^{2}(0, T)$ and $f \in L^{2}\left(0, T ; L^{2}(0, L)\right)$, there is a unique solution $v \in C\left([0, T] ; H^{1}(0, L)\right) \cap C^{1}\left([0, T] ; L^{2}(0, L)\right)$ of

$$
\begin{array}{cc}
v_{t t}-v_{x x}+\alpha v_{t}=f \quad \text { for }(x, t) \in(0, L) \times(0, T), \\
v_{x}(0, t)=q(t), \quad v(L, t)=0 \quad \text { for } t \in(0, T), \\
v(x, 0)=0, \quad v_{t}(x, 0)=0 \quad \text { for } x \in(0, L) .
\end{array}
$$

Furthermore, it holds that

$$
\begin{aligned}
& \frac{1}{2}\left\|v_{t}(t)\right\|_{L^{2}(0, L)}^{2}+\frac{1}{2}\left\|v_{x}(t)\right\|_{L^{2}(0, L)}^{2} \\
& =-\int_{0}^{t} v_{s}(0, s) q(s) d s-\alpha \int_{0}^{t}\left\|v_{s}(s)\right\|_{L^{2}(0, L)}^{2} d s+\int_{0}^{t} \int_{0}^{L} f v_{s} d x d s
\end{aligned}
$$

for all $t \in[0, T]$,

$$
\left\|v_{t}(t)\right\|_{L^{2}(0, L)}^{2}+\|v(t)\|_{H^{1}(0, L)}^{2} \leq M \int_{0}^{t}|q(s)|^{2} d s+M \int_{0}^{t}\|f(s)\|_{L^{2}(0, L)}^{2} d s
$$
for all $t \in[0, T]$, and

$$
\begin{aligned}
& \int_{0}^{t}\left|v_{s}(0, s)\right|^{2} d s+\int_{0}^{t}|q(s)|^{2} d s \leq M\left(\left\|v_{t}(t)\right\|_{L^{2}(0, L)}^{2}+\|v(t)\|_{H^{1}(0, L)}^{2}\right) \\
& +M \int_{0}^{t}\left(\left\|v_{s}(s)\right\|_{L^{2}(0, L)}^{2}+\|v(s)\|_{H^{1}(0, L)}^{2}\right) d s+M \int_{0}^{t}\|f(s)\|_{L^{2}(0, L)}^{2} d s
\end{aligned}
$$

for all $t \in[0, T]$. Here $M$ denotes positive constants independent of $q$ and $f$.

For the proof, see Proposition 1.5 of [9], where a different boundary condition was used at $x=L$, but the details of the proof are essentially the same. We note that if $q=q_{m} \in C^{3}([0, T])$ and $f=f_{m} \in C^{1}\left([0, T] ; L^{2}(0, L)\right)$, then the corresponding solution $v_{m}$ satisfies additional regularity such that

$$
\begin{aligned}
& v_{m} \in L^{\infty}\left(0, T ; H^{2}(0, L)\right), \quad \partial_{t} v_{m} \in L^{\infty}\left(0, T ; H^{1}(0, L)\right), \\
& \partial_{t t} v_{m} \in L^{\infty}\left(0, T ; L^{2}(0, L)\right) .
\end{aligned}
$$

If $q_{m} \rightarrow q$ in $L^{2}(0, T)$ and $f_{m} \rightarrow f$ in $L^{2}\left(0, T ; L^{2}(0, L)\right)$, then (2.11) and (2.12) imply that

$$
v_{m} \rightarrow v \quad \text { in } C\left([0, T] ; H^{1}(0, L)\right) \cap C^{1}\left([0, T] ; L^{2}(0, L)\right)
$$

and

$$
\partial_{t} v_{m}(0, \cdot) \rightarrow \partial_{t} v(0, \cdot) \quad \text { in } L^{2}(0, T)
$$

Lemma 2.3. For given $h \in H^{1}(0, T)$, there is a unique solution $v \in C([0, T]$; $\left.H^{1}(0, L)\right) \cap C^{1}\left([0, T] ; L^{2}(0, L)\right)$ of

$$
\begin{gathered}
v_{t t}-v_{x x}+\alpha v_{t}=0 \quad \text { for }(x, t) \in(0, L) \times(0, T), \\
v_{x}(0, t)=-K(v(0, t)+h(t))^{-}, \quad v(L, t)=0 \quad \text { for } t \in(0, T), \\
v(x, 0)=0, \quad v_{t}(x, 0)=0 \quad \text { for } x \in(0, L)
\end{gathered}
$$


where $K$ is a positive constant. Furthermore, if $h(0) \geq 0$, it holds that

$$
\begin{aligned}
& \left\|v_{t}(t)\right\|_{L^{2}(0, L)}^{2}+\|v(t)\|_{H^{1}(0, L)}^{2}+K\left|(v(0, t)+h(t))^{-}\right|^{2} \\
& \quad+\int_{0}^{t}\left|v_{x}(0, s)\right|^{2} d s+\int_{0}^{t}\left|v_{s}(0, s)\right|^{2} d s \leq M \int_{0}^{t}\left|h_{s}(s)\right|^{2} d s
\end{aligned}
$$

for all $t \in[0, T]$, for some constant $M$ independent of $K$ and $h$. If $v_{1}$ and $v_{2}$ are solutions corresponding to $h=h_{1}$ and $h=h_{2}$, respectively, it holds that

$$
\begin{aligned}
& \left\|\partial_{t} v_{1}(t)-\partial_{t} v_{2}(t)\right\|_{L^{2}(0, L)}^{2}+\left\|v_{1}(t)-v_{2}(t)\right\|_{H^{1}(0, L)}^{2} \\
& +\int_{0}^{t}\left|\partial_{s} v_{1}(0, s)-\partial_{s} v_{2}(0, s)\right|^{2} d s+\int_{0}^{t}\left|\partial_{x} v_{1}(0, s)-\partial_{x} v_{2}(0, s)\right|^{2} d s \\
& \leq M_{K} \int_{0}^{t}\left|h_{1}(s)-h_{2}(s)\right|^{2} d s
\end{aligned}
$$

for all $t \in[0, T]$, for some constant $M_{K}$ independent of $h_{1}$ and $h_{2}$.

Proof. Existence of a solution follows from Lemma 2.2 and an iteration scheme.

For (2.19), we find from (2.10) that

$$
\begin{aligned}
& \left\|v_{t}(t)\right\|_{L^{2}(0, L)}^{2}+\left\|v_{x}(t)\right\|_{L^{2}(0, L)}^{2}=2 \int_{0}^{t} K(v(0, s)+h(s))^{-}\left(v_{s}(0, s)+h_{s}(s)\right) d s \\
& -2 \int_{0}^{t} K(v(0, s)+h(s))^{-} h_{s}(s) d s-2 \alpha \int_{0}^{t}\left\|v_{s}(s)\right\|_{L^{2}(0, L)}^{2} d s
\end{aligned}
$$

for all $t \in[0, T]$. In the meantime, it follows from (2.12) that

$$
\begin{aligned}
& \int_{0}^{t}\left(K(v(0, s)+h(s))^{-}\right)^{2} d s+\int_{0}^{t}\left|v_{s}(0, s)\right|^{2} d s \\
& \quad \leq M\left(\left\|v_{t}(t)\right\|_{L^{2}(0, L)}^{2}+\|v(t)\|_{H^{1}(0, L)}^{2}\right) \\
& \quad+M \int_{0}^{t}\left(\left\|v_{s}(s)\right\|_{L^{2}(0, L)}^{2}+\|v(s)\|_{H^{1}(0, L)}^{2}\right) d s .
\end{aligned}
$$

Since $v(0,0)+h(0) \geq 0$, it follows from (1.1) that

$$
2 \int_{0}^{t} K(v(0, s)+h(s))^{-}\left(v_{s}(0, s)+h_{s}(s)\right) d s=-K\left|(v(0, t)+h(t))^{-}\right|^{2} .
$$

We now use (2.23) and

$$
\begin{aligned}
& -2 \int_{0}^{t} K(v(0, s)+h(s))^{-} h_{s}(s) d s \leq \epsilon \int_{0}^{t}\left(K(v(0, s)+h(s))^{-}\right)^{2} d s \\
& +\frac{1}{\epsilon} \int_{0}^{t}\left|h_{s}(s)\right|^{2} d s, \quad \text { for each } \epsilon>0
\end{aligned}
$$

to derive (2.19) from (2.21) and (2.22) with help of the Gronwall inequality.

Next we use (2.11) and (2.12) with $q=-K\left(v_{1}(0, t)+h_{1}(t)\right)^{-}+K\left(v_{2}(0, t)+\right.$ $\left.h_{2}(t)\right)^{-}, v=v_{1}-v_{2}$ and $f \equiv 0$. By $(2.11)$ and

$$
\int_{0}^{t}\left|v_{1}(0, s)-v_{2}(0, s)\right|^{2} d s \leq M \int_{0}^{t}\left\|v_{1}(s)-v_{2}(s)\right\|_{H^{1}(0, L)}^{2} d s,
$$


we can again use the Gronwall inequality to derive

$$
\begin{aligned}
& \left\|\partial_{t} v_{1}(t)-\partial_{t} v_{2}(t)\right\|_{L^{2}(0, L)}^{2}+\left\|v_{1}(t)-v_{2}(t)\right\|_{H^{1}(0, L)}^{2} \\
& \leq M_{K} \int_{0}^{t}\left|h_{1}(s)-h_{2}(s)\right|^{2} d s
\end{aligned}
$$

for all $t \in[0, T]$, for some constant $M_{K}$ independent of $h_{1}$ and $h_{2}$.

This, combined with (2.12), yields (2.20).

\section{§3. Stochastic EQUation}

Let us consider the initial-boundary value problem

$$
\begin{gathered}
w_{t t}-w_{x x}+\alpha w_{t}=f+\frac{\partial}{\partial t} \sum_{j=1}^{\infty} \int_{0}^{t} g_{j} d B_{j} \quad \text { for }(x, t) \in(0, L) \times(0, T), \\
w_{x}(0, t)=0, \quad w(L, t)=0 \quad \text { for } 0<t<T, \\
w(x, 0)=u_{0}(x), \quad w_{t}(x, 0)=u_{1}(x) \quad \text { for } 0<x<L .
\end{gathered}
$$

Here $f$ and the $g_{j}$ 's are given functions such that they are progressively measurable, $f \in L^{2}\left(\Omega ; L^{2}\left(0, T ; L^{2}(0, L)\right)\right)$, for each $T>0$, and

$$
\sum_{j=1}^{\infty} E\left(\left\|g_{j}\right\|_{L^{2}\left(0, T ; L^{2}(0, L)\right)}^{2}\right)<\infty
$$

for each $T>0$.

Lemma 3.1. Suppose that $u_{0}$ and $u_{1}$ are $\mathcal{F}_{0}$-measurable such that $u_{1} \in L^{2}(\Omega$; $\left.L^{2}(0, L)\right)$ and $u_{0} \in L^{2}\left(\Omega ; H^{1}(0, L)\right)$ with $u_{0}(L)=0$, for almost all $\omega \in \Omega$. Then, there is a unique solution $w$ of (3.1) - (3.3) such that $w$ is progressively measurable and

$$
w \in L^{2}\left(\Omega ; C\left([0, T] ; H^{1}(0, L)\right) \cap C^{1}\left([0, T] ; L^{2}(0, L)\right)\right)
$$

for each $T>0$. Furthermore, it holds that

$$
\begin{aligned}
& E\left(\sup _{s \in[0, t]}\|w(s)\|_{H^{1}(0, L)}^{2}\right)+E\left(\sup _{s \in[0, t]}\left\|w_{s}(s)\right\|_{L^{2}(0, L)}^{2}\right)+E\left(\int_{0}^{t}\left|w_{s}(0, s)\right|^{2} d s\right) \\
& \leq M E\left(\left\|u_{0}\right\|_{H^{1}(0, L)}^{2}\right)+M E\left(\left\|u_{1}\right\|_{L^{2}(0, L)}^{2}\right) \\
& \quad+M E\left(\int_{0}^{t}\|f\|_{L^{2}(0, L)}^{2} d s\right)+M E\left(\sum_{j=1}^{\infty} \int_{0}^{t}\left\|g_{j}\right\|_{L^{2}(0, L)}^{2} d s\right)
\end{aligned}
$$

for all $t \in[0, T]$, for some positive constant $M$ depending only on $T>0$.

In order to justify manipulations to obtain (3.6), we need more regularity than is indicated by (3.5). Therefore, we first consider the case of more regular data.

Let $\left\{e_{k}\right\}_{k=1}^{\infty}$ be a complete orthonormal basis for $L^{2}(0, L)$ such that

$$
\left\{\begin{array}{l}
-\partial_{x x} e_{k}=\lambda_{k} e_{k}, \quad \text { for } x \in(0, L), \\
\partial_{x} e_{k}(0)=0, \quad e_{k}(L)=0
\end{array}\right.
$$

We write

$$
\Psi(t)=\sum_{j=1}^{\infty} \int_{0}^{t} g_{j}(s) d B_{j}(s),
$$


and define for each $\nu \geq 1$,

$$
\begin{array}{rlrl}
u_{0, \nu} & =\sum_{k=1}^{\nu}\left\langle u_{0}, e_{k}\right\rangle e_{k}, & u_{1, \nu} & =\sum_{k=1}^{\nu}\left\langle u_{1}, e_{k}\right\rangle e_{k}, \\
f_{\nu} & =\sum_{k=1}^{\nu}\left\langle f, e_{k}\right\rangle e_{k}, \quad g_{j, \nu}=\sum_{k=1}^{\nu}\left\langle g_{j}, e_{k}\right\rangle e_{k}
\end{array}
$$

and

$$
\Psi_{\nu}(t)=\sum_{j=1}^{\infty} \int_{0}^{t} g_{j, \nu}(s) d B_{j}(s) .
$$

Here, $\langle\cdot, \cdot\rangle$ denotes the inner product in $L^{2}(0, L)$.

Lemma 3.2. Fix any $T>0$ and $\nu \geq 1$. For $u_{0, \nu}$ and $u_{1, \nu}$ defined as above, there is a unique solution $w_{\nu}$ of

$$
\begin{gathered}
\partial_{t t} w_{\nu}-\partial_{x x} w_{\nu}+\alpha \partial_{t} w_{\nu}=f_{\nu}+\partial_{t} \Psi_{\nu}, \quad \text { for }(x, t) \in(0, L) \times(0, T), \\
\partial_{x} w_{\nu}(0, t)=0, \quad w_{\nu}(L, t)=0, \quad \text { for } t \in(0, T), \\
w_{\nu}(x, 0)=u_{0, \nu}(x), \quad \partial_{t} w_{\nu}(x, 0)=u_{1, \nu}(x), \quad \text { for } x \in(0, L)
\end{gathered}
$$

such that $w_{\nu}$ is progressively measurable and

$$
w_{\nu} \in L^{2}\left(\Omega ; C^{1}\left([0, T] ; H^{m}(0, L)\right)\right), \quad \text { for all } m \geq 1 .
$$

Furthermore, it holds that

$$
\begin{gathered}
E\left(\sup _{s \in[0, t]}\left\|w_{\nu}(s)\right\|_{H^{1}(0, L)}^{2}\right)+E\left(\sup _{s \in[0, t]}\left\|\partial_{s} w_{\nu}(s)\right\|_{L^{2}(0, L)}^{2}\right) \\
+E\left(\int_{0}^{t}\left|\partial_{s} w_{\nu}(0, s)\right|^{2} d s\right) \leq M E\left(\left\|u_{0, \nu}\right\|_{H^{1}(0, L)}^{2}\right)+M E\left(\left\|u_{1, \nu}\right\|_{L^{2}(0, L)}^{2}\right) \\
+M E\left(\int_{0}^{t}\left\|f_{\nu}(s)\right\|_{L^{2}(0, L)}^{2} d s\right) \\
+M E\left(\sum_{j=1}^{\infty} \int_{0}^{t}\left\|g_{j, \nu}(s)\right\|_{L^{2}(0, L)}^{2} d s\right)
\end{gathered}
$$

for all $t \in[0, T]$, for some positive constant $M$ depending only on $T>0$.

Proof. It is easy to see that a solution $w_{\nu}$ can be represented by

$$
w_{\nu}(x, t)=\sum_{k=1}^{\nu} c_{k}(t) e_{k}(x)
$$

where the $c_{k}$ 's satisfy the system of stochastic differential equations

$$
\partial_{t t} c_{k}=-\lambda_{k} c_{k}-\alpha \partial_{t} c_{k}+\left\langle f_{\nu}+\partial_{t} \Psi_{\nu}, e_{k}\right\rangle, \quad 1 \leq k \leq \nu,
$$

and the initial conditions

$$
c_{k}(0)=\left\langle u_{0}, e_{k}\right\rangle, \quad \partial_{t} c_{k}(0)=\left\langle u_{1}, e_{k}\right\rangle, \quad 1 \leq k \leq \nu .
$$

We can write (3.8) as

$$
d\left(\partial_{t} w_{\nu}\right)=\left(\partial_{x x} w_{\nu}-\alpha \partial_{t} w_{\nu}\right) d t+f_{\nu} d t+d \Psi_{\nu}
$$


By Ito's rule and integration by parts using (3.9), we have

$$
\begin{aligned}
\left\|\partial_{t} w_{\nu}(t)\right\|_{L^{2}(0, L)}^{2}+\left\|\partial_{x} w_{\nu}(t)\right\|_{L^{2}(0, L)}^{2}=\left\|u_{1, \nu}\right\|_{L^{2}(0, L)}^{2}+\left\|\partial_{x} u_{0, \nu}\right\|_{L^{2}(0, L)}^{2} & \\
& -2 \alpha \int_{0}^{t}\left\|\partial_{s} w_{\nu}\right\|_{L^{2}(0, L)}^{2} d s+2 \int_{0}^{t}\left\langle f_{\nu}, \partial_{s} w_{\nu}\right\rangle d s \\
& +2 \sum_{j=1}^{\infty} \int_{0}^{t}\left\langle\partial_{s} w_{\nu}, g_{j, \nu}\right\rangle d B_{j}(s)+\sum_{j=1}^{\infty} \int_{0}^{t}\left\|g_{j, \nu}\right\|_{L^{2}(0, L)}^{2} d s
\end{aligned}
$$

for all $t \in[0, T]$, for almost all $\omega \in \Omega$. By the Burkholder-Davis-Gundy inequality,

$$
\begin{aligned}
& E\left(\sup _{s \in[0, t]}\left|\sum_{j=1}^{\infty} \int_{0}^{s}\left\langle\partial_{\eta} w_{\nu}, g_{j, \nu}\right\rangle d B_{j}(\eta)\right|\right) \\
& \leq C E\left(\sum_{j=1}^{\infty} \int_{0}^{t}\left\|\partial_{s} w_{\nu}\right\|_{L^{2}(0, L)}^{2}\left\|g_{j, \nu}\right\|_{L^{2}(0, L)}^{2} d s\right)^{1 / 2} \\
& \leq \delta E\left(\sup _{s \in[0, t]}\left\|\partial_{s} w_{\nu}(s)\right\|_{L^{2}(0, L)}^{2}\right)+\frac{C^{2}}{\delta} E\left(\sum_{j=1}^{\infty} \int_{0}^{t}\left\|g_{j, \nu}\right\|_{L^{2}(0, L)}^{2} d s\right)
\end{aligned}
$$

for all $\delta>0$, for some positive constant $C$ independent of $\nu$.

Hence, it follows from (3.17) that

$$
\begin{aligned}
& E\left(\sup _{s \in[0, t]}\left\|\partial_{s} w_{\nu}(s)\right\|_{L^{2}(0, L)}^{2}+\sup _{s \in[0, t]}\left\|w_{\nu}(s)\right\|_{H^{1}(0, L)}^{2}\right) \\
& \leq M E\left(\left\|u_{0, \nu}\right\|_{H^{1}(0, L)}^{2}\right)+M E\left(\left\|u_{1, \nu}\right\|_{L^{2}(0, L)}^{2}\right) \\
& +M E\left(\int_{0}^{t}\left\|f_{\nu}\right\|_{L^{2}(0, L)} d s\right)^{2}+M \sum_{j=1}^{\infty} E\left(\int_{0}^{t}\left\|g_{j, \nu}\right\|_{L^{2}(0, L)}^{2} d s\right)
\end{aligned}
$$

for all $t \in[0, T]$, for some positive constant $M$ independent of $\nu$. Next let $\psi \in$ $C^{1}([0, L])$ such that $\psi(0)=1$ and $\psi(L)=\psi_{x}(L)=0$, and write

$$
d\left(\psi \partial_{x} w_{\nu}\right)=\left(\psi \partial_{x t} w_{\nu}\right) d t
$$

By applying Ito's rule to the functional $\left\langle\partial_{t} w_{\nu}, \psi \partial_{x} w_{\nu}\right\rangle$, we find

$$
\begin{aligned}
& \left\langle\partial_{t} w_{\nu}(t), \psi \partial_{x} w_{\nu}(t)\right\rangle-\left\langle u_{1, \nu}, \psi \partial_{x} u_{0, \nu}\right\rangle=-\frac{1}{2} \int_{0}^{t}\left|\partial_{s} w_{\nu}(0, s)\right|^{2} d s \\
& -\int_{0}^{t} \int_{0}^{L}\left(\frac{1}{2} \psi_{x}\left(\partial_{x} w_{\nu}\right)^{2}+\alpha \psi\left(\partial_{x} w_{\nu}\right) \partial_{s} w_{\nu}+\frac{1}{2} \psi_{x}\left(\partial_{s} w_{\nu}\right)^{2}\right) d x d s \\
& +\int_{0}^{t}\left\langle\psi \partial_{x} w_{\nu}, f_{\nu}\right\rangle d s+\sum_{j=1}^{\infty} \int_{0}^{t}\left\langle\psi \partial_{x} w_{\nu}, g_{j, \nu}\right\rangle d B_{j}(s)
\end{aligned}
$$

for all $t \in[0, T]$, for almost all $\omega \in \Omega$. By taking the expectation of (3.21) and combining it with (3.19), we obtain (3.12). The argument for the pathwise uniqueness of solutions is the same as that for the deterministic equation.

Proof of Lemma 3.1. Let $u_{0} \in H^{1}(0, L)$ with $u_{0}(L)=0$, and $u_{1} \in L^{2}(0, L)$ be given. We define $u_{0, \nu}$ and $u_{1, \nu}$ as above. Then, as $\nu \rightarrow \infty, u_{0, \nu} \rightarrow u_{0}$ in $H^{1}(0, L)$, 
and $u_{1, \nu} \rightarrow u_{1}$ in $L^{2}(0, L)$. Let $w_{\nu}$ be the solution of (3.8) - (3.10). We also note that as $\nu \rightarrow \infty$,

$$
E\left(\sum_{j=1}^{\infty} \int_{0}^{T}\left\|g_{j, \nu}-g_{j}\right\|_{L^{2}(0, L)}^{2} d t\right) \rightarrow 0 .
$$

We can estimate $w_{\nu_{1}}-w_{\nu_{2}}$ in the same way as for (3.12) and find that as $\nu \rightarrow \infty$,

$$
w_{\nu} \rightarrow w \quad \text { in } L^{2}\left(\Omega ; C\left([0, T] ; H^{1}(0, L)\right)\right)
$$

and

$$
\partial_{t} w_{\nu} \rightarrow \partial_{t} w \quad \text { in } L^{2}\left(\Omega ; C\left([0, T] ; L^{2}(0, L)\right)\right)
$$

where $w$ is a solution of

$$
\left\{\begin{array}{l}
\partial_{t t} w-\partial_{x x} w+\alpha \partial_{t} w=f+\partial_{t} \Psi \quad \text { for }(x, t) \in(0, L) \times(0, T), \\
w(L, t)=0 \quad \text { for } t \in(0, T), \\
w(x, 0)=u_{0}(x), \quad \partial_{t} w(x, 0)=u_{1}(x), \quad \text { for } x \in(0, L) .
\end{array}\right.
$$

Obviously $w$ is progressively measurable. Next we show that the trace of $w_{t}$ and $w_{x}$ at $x=0$ is well defined and belongs to $L^{2}\left(\Omega ; L^{2}(0, T)\right)$. For almost all $\omega \in \Omega$, it holds that

$$
\partial_{x x} w_{\nu}=\partial_{t t} w_{\nu}+\alpha \partial_{t} w_{\nu}-f_{\nu}-\partial_{t} \Psi_{\nu}
$$

and

$$
\partial_{x x} w=\partial_{t t} w+\alpha w_{t}-f-\partial_{t} \Psi
$$

in the sense of distributions over $(0, L) \times(0, T)$. Since $\Psi_{\nu} \rightarrow \Psi$ in $L^{2}\left(\Omega ; L^{2}((0, L) \times\right.$ $(0, T)))$ as $\nu \rightarrow \infty$, it follows from $(3.23)-(3.27)$ that

$$
\partial_{x x} w_{\nu} \rightarrow \partial_{x x} w \quad \text { in } L^{2}\left(\Omega ; L^{2}\left(0, L ; H^{-1}(0, T)\right)\right) \text { as } \nu \rightarrow \infty .
$$

Consequently, as $\nu \rightarrow \infty$,

$$
\partial_{x} w_{\nu} \rightarrow \partial_{x} w \quad \text { in } L^{2}\left(\Omega ; C\left([0, L] ; H^{-1}(0, T)\right)\right)
$$

and

$$
\partial_{t} w_{\nu} \rightarrow \partial_{t} w \quad \text { in } L^{2}\left(\Omega ; C\left([0, L] ; H^{-2}(0, T)\right)\right) .
$$

Thus, it follows that

$$
\partial_{t} w_{\nu}(0, \cdot) \rightarrow \partial_{t} w(0, \cdot) \quad \text { in } L^{2}\left(\Omega ; H^{-2}(0, T)\right)
$$

and

$$
\partial_{x} w_{\nu}(0, \cdot) \rightarrow \partial_{x} w(0, \cdot) \quad \text { in } L^{2}\left(\Omega ; H^{-1}(0, T)\right) .
$$

By means of (3.12), it must hold that

$$
\partial_{t} w_{\nu}(0, \cdot) \rightarrow \partial_{t} w(0, \cdot) \quad \text { weakly in } L^{2}\left(\Omega ; L^{2}(0, T)\right) .
$$

For each $\nu \geq 1, \partial_{t} w_{\nu}(0, \cdot)$ is progressively measurable and hence, $\partial_{t} w(0, \cdot)$ is also progressively measurable. Since $\partial_{x} w_{\nu}(0, \cdot) \equiv 0$, for all $\nu$, we also have

$$
\partial_{x} w(0, \cdot) \equiv 0 \text {. }
$$

Hence, $w$ is a solution of (3.1) - (3.3), and (3.6) follows from (3.12), (3.23), (3.24) and (3.31). The pathwise uniqueness of solution of (3.1) - (3.3) follows from the well-known uniqueness result on the deterministic equation. 
Lemma 3.3. Let $f$ be progressively measurable such that $f \in L^{1}\left(\Omega ; L^{1}(0, T)\right)$. Suppose that $v \in L^{2}\left(\Omega ; C\left([0, T] ; H^{1}(0, L)\right) \cap C^{1}\left([0, T] ; L^{2}(0, L)\right)\right)$ is progressively measurable and is a solution of

$$
\begin{gathered}
v_{t t}-v_{x x}+\alpha v_{t}=\sum_{j=1}^{\infty} \sigma_{j} v \frac{d B_{j}}{d t} \quad \text { for }(x, t) \in(0, L) \times(0, T), \\
v_{x}(0, t) v(0, t) \geq-|f(t)| \quad \text { for } t \in(0, T), \\
v(L, t)=0 \quad \text { for } t \in(0, T), \\
v(x, 0)=0, \quad v_{t}(x, 0)=0 \quad \text { for } x \in(0, L) .
\end{gathered}
$$

Then, there is some $0<\delta<T$ and a positive constant $M_{\delta}$ independent of $v$, the $\sigma_{j}$ 's and $f$ such that

$$
E\left(\sup _{t \in[0, \delta]}|v(0, t)|^{2}\right) \leq M_{\delta} E\left(\int_{0}^{\delta}|f(s)| d s\right) .
$$

Proof. Let $\tilde{v}=v e^{\alpha t / 2}$. Then, $\tilde{v}$ satisfies the equation

$$
v_{t t}-v_{x x}=\frac{\alpha^{2}}{4} v+\sum_{j=1}^{\infty} \sigma_{j} v \frac{d B_{j}}{d t}, \quad \text { for }(x, t) \in(0, L) \times(0, T)
$$

and (3.34) - (3.36) with $f(t)$ replaced by $f(t) e^{\alpha t}$. Hence, we may consider (3.38) instead of (3.33). Now suppose that $v$ is a solution of (3.34) - (3.36) and (3.38).

With this $v$ as a given function, let $\phi \in L^{2}\left(\Omega ; C\left([0, T] ; H^{1}(0, L)\right) \cap C^{1}([0, T]\right.$; $\left.\left.L^{2}(0, L)\right)\right)$ be the unique solution of

$$
\left\{\begin{array}{l}
\phi_{t t}-\phi_{x x}=\frac{\alpha^{2}}{4} v+\sum_{j=1}^{\infty} \sigma_{j} v \frac{d B_{j}}{d t} \quad \text { for }(x, t) \in(0, L) \times(0, T), \\
\phi_{x}(0, t)=0, \quad \phi(L, t)=0 \quad \text { for } t \in(0, T), \\
\phi(x, 0)=0, \quad \phi_{t}(x, 0)=0 \quad \text { for } x \in(0, L),
\end{array}\right.
$$

and let $\psi=v-\phi$. Then, $\psi \in C\left([0, T] ; H^{1}(0, L)\right) \cap C^{1}\left([0, T] ; L^{2}(0, L)\right)$, for almost all $\omega \in \Omega$, and $\psi$ satisfies

$$
\left\{\begin{array}{l}
\psi_{t t}-\psi_{x x}=0 \quad \text { for }(x, t) \in(0, L) \times(0, T) \\
\psi(L, t)=0 \quad \text { for } t \in(0, T) \\
\psi(x, 0)=0, \quad \psi_{t}(x, 0)=0 \quad \text { for } x \in(0, L) .
\end{array}\right.
$$

There is some $\tilde{\Omega} \subset \Omega$ such that $P(\Omega \backslash \tilde{\Omega})=0$ and for each $\omega \in \tilde{\Omega}$, (3.34) - (3.36) and (3.38) - (3.40) hold. Let us fix any $\omega \in \tilde{\Omega}$. For all $(x, t)$ such that $0 \leq t \leq \min (T, L / 2), 0 \leq x \leq L / 2, t \geq x$, it holds that

$$
\psi(x, t)+\psi\left(\frac{t-x}{2}, \frac{t-x}{2}\right)=\psi(0, t-x)+\psi\left(\frac{t+x}{2}, \frac{t+x}{2}\right) .
$$

For this identity, see John [6]. In the meantime, by the domain of dependence,

$$
\psi\left(\frac{t-x}{2}, \frac{t-x}{2}\right)=\psi\left(\frac{t+x}{2}, \frac{t+x}{2}\right)=0 .
$$


Hence,

$$
\psi(x, t)=\psi(0, t-x)
$$

By the same argument, for all $(x, t)$ such that $L / 2 \leq x \leq L, 0 \leq t \leq \min (T, L / 2)$, $x+t \geq L$,

$$
\psi(x, t)=\psi(L, x+t-L) .
$$

Meanwhile, $\psi(L, t)=0$, for all $0 \leq t \leq T$, and hence, it follows that, for all $(x, t)$ such that $L / 2 \leq x \leq L, 0 \leq t \leq \min (T, L / 2), x+t \geq L$,

$$
\psi(x, t)=0 .
$$

Again by the domain of dependence, (3.43) is also valid for all $(x, t)$ such that $0 \leq x \leq L, 0 \leq t \leq \min (T, L / 2), t \leq x$ and $t \leq L-x$. We see from (3.41) that

$$
\psi_{t}(0, t)=-\psi_{x}(0, t)
$$

for almost all $t \in(0, \min (T, L / 2))$. Since $\psi_{x}(0, t)=v_{x}(0, t)$, it follows that

$$
v(0, t)\left(v_{t}(0, t)-\phi_{t}(0, t)\right)=v(0, t) \psi_{t}(0, t) \leq|f(t)|
$$

for almost all $t \in(0, \min (T, L / 2))$, and thus,

$$
|v(0, t)|^{2} \leq \int_{0}^{t}|v(0, s)|^{2} d s+\int_{0}^{t}\left|\phi_{s}(0, s)\right|^{2} d s+2 \int_{0}^{t}|f(s)| d s
$$

for all $t \in[0, \min (T, L / 2)]$. This is true for each $\omega \in \tilde{\Omega}$. In the meantime, it follows from Lemma 3.1 that

$$
\begin{aligned}
& E\left(\sup _{s \in[0, t]}\|\phi(s)\|_{H^{1}(0, L)}^{2}\right)+E\left(\sup _{s \in[0, t]}\left\|\phi_{s}(s)\right\|_{L^{2}(0, L)}^{2}\right)+E\left(\int_{0}^{t}\left|\phi_{s}(0, s)\right|^{2} d s\right) \\
& \leq \operatorname{ME}\left(\int_{0}^{t} \int_{0}^{L}|v|^{2} d x d s\right)
\end{aligned}
$$

and

$$
\begin{aligned}
& E\left(\int_{0}^{t} \int_{0}^{L}|v|^{2} d x d s\right) \leq 2 E\left(\int_{0}^{t} \int_{0}^{L}\left(|\psi|^{2}+|\phi|^{2}\right) d x d s\right) \\
& \leq 2 E\left(\int_{0}^{t} \int_{0}^{L}|\psi|^{2} d x d s\right)+2 t^{2} E\left(\int_{0}^{t} \int_{0}^{L}\left|\phi_{s}\right|^{2} d x d s\right) \\
& \leq 2 E\left(\int_{0}^{t} \int_{0}^{L}|\psi|^{2} d x d s\right)+M t^{2} E\left(\int_{0}^{t} \int_{0}^{L}|v|^{2} d x d s\right)
\end{aligned}
$$

for all $t \in[0, T]$, for some positive constant $M$ independent of $v$. Thus, there is some $0<\delta<\min (T, L / 2)$ independent of $v$ and a positive constant $M_{\delta}$ depending on $\delta$ such that

$$
E\left(\int_{0}^{t} \int_{0}^{L}|v|^{2} d x d s\right) \leq M_{\delta} E\left(\int_{0}^{t} \int_{0}^{L}|\psi|^{2} d x d s\right),
$$

for all $0 \leq t \leq \delta$. Hence, it follows from (3.47) that

$$
E\left(\int_{0}^{t}\left|\phi_{s}(0, s)\right|^{2} d s\right) \leq M_{\delta} E\left(\int_{0}^{t} \int_{0}^{L}|\psi|^{2} d x d s\right)
$$


for all $0 \leq t \leq \delta$. In the meantime, we derive from (3.41) and (3.43) that

$$
\begin{aligned}
& E\left(\int_{0}^{t} \int_{0}^{L}|\psi(x, s)|^{2} d x d s\right)=E\left(\int_{0}^{t} \int_{0}^{s}|\psi(0, s-x)|^{2} d x d s\right) \\
& \leq t E\left(\int_{0}^{t}|\psi(0, s)|^{2} d s\right) \\
& \leq 2 t E\left(\int_{0}^{t}|v(0, s)|^{2} d s\right)+2 t E\left(\int_{0}^{t}|\phi(0, s)|^{2} d s\right) \\
& \leq 2 t E\left(\int_{0}^{t}|v(0, s)|^{2} d s\right)+2 t^{3} E\left(\int_{0}^{t}\left|\phi_{s}(0, s)\right|^{2} d s\right) .
\end{aligned}
$$

By taking $\delta$ smaller if necessary, we find that for all $0 \leq t \leq \delta$,

$$
E\left(\int_{0}^{t}\left|\phi_{s}(0, s)\right|^{2} d s\right) \leq M_{\delta} E\left(\int_{0}^{t}|v(0, s)|^{2} d s\right),
$$

which, together with (3.46), yields

$$
E\left(\sup _{s \in[0, t]}|v(0, s)|^{2}\right) \leq M_{\delta} \int_{0}^{t} E\left(|v(0, s)|^{2}\right) d s+2 E\left(\int_{0}^{t}|f(s)| d s\right)
$$

for all $0 \leq t \leq \delta$. By the Gronwall inequality, we have (3.37).

Remark 3.4. If $f \equiv 0$ in (3.34), then $v(0, t)=0$ for all $t \in[0, \delta]$, for almost all $\omega$, and thus, $v \equiv 0$ on $[0, \delta]$ for almost all $\omega$. By repetition, we conclude that $v \equiv 0$ on $[0, T]$, for almost all $\omega$.

\section{§4. Proof of Theorem 1.4}

Let us choose any $T>0$. According to Lemma 3.1, let $w \in L^{2}(\Omega ; C([0, T]$; $\left.\left.H^{1}(0, L)\right) \cap C^{1}\left([0, T] ; L^{2}(0, L)\right)\right)$ be a unique solution of

$$
\left\{\begin{array}{l}
w_{t t}-w_{x x}+\alpha w_{t}=\sum_{j=1}^{\infty} g_{j} \frac{d B_{j}}{d t} \quad \text { for }(x, t) \in(0, L) \times(0, T), \\
w_{x}(0, t)=0, \quad w(L, t)=0 \text { for } t \in(0, T) \\
w(x, 0)=u_{0}(x), \quad w_{t}(x, 0)=u_{1}(x) \quad \text { for } x \in(0, L)
\end{array}\right.
$$

Let $v$ be the unique solution in Lemma 2.3 with

$$
h(t)=w(0, t) .
$$

By virtue of (2.20), the mapping

$$
h \mapsto v
$$

is continuous from $L^{2}(0, T)$ into $C\left([0, T] ; H^{1}(0, L)\right) \cap C^{1}\left([0, T] ; L^{2}(0, L)\right)$. Also, we find that if $h \in L^{2}\left(\Omega ; L^{2}(0, T)\right)$, then $v \in L^{2}\left(\Omega ; C\left([0, T] ; H^{1}(0, L)\right) \cap C^{1}([0, T]\right.$; $\left.\left.L^{2}(0, L)\right)\right)$. At the same time, if $h$ is progressively measurable, so is $v$. Next we write

$$
u=w+v .
$$


Then, $u \in L^{2}\left(\Omega ; C\left([0, T] ; H^{1}(0, L)\right) \cap C^{1}\left([0, T] ; L^{2}(0, L)\right)\right)$ is a unique solution of

$$
\left\{\begin{array}{l}
u_{t t}-u_{x x}+\alpha u_{t}=\sum_{j=1}^{\infty} g_{j} \frac{d B_{j}}{d t} \quad \text { for }(x, t) \in(0, L) \times(0, T), \\
u_{x}(0, t)=-K u(0, t)^{-}, \quad u(L, t)=0 \quad \text { for } t \in(0, T), \\
u(x, 0)=u_{0}(x), \quad u_{t}(x, 0)=u_{1}(x) \quad \text { for } x \in(0, L)
\end{array}\right.
$$

Let $u$ and $\tilde{u}$ be the solutions corresponding to $\left\{g_{j}\right\}_{j=1}^{\infty}$ and $\left\{\tilde{g}_{j}\right\}_{j=1}^{\infty}$, respectively. Then, we can write

$$
u=w+v, \quad \tilde{u}=\tilde{w}+\tilde{v}
$$

where $w$ and $\tilde{w}$ are solutions of (4.1) corresponding to $\left\{g_{j}\right\}_{j=1}^{\infty}$ and $\left\{\tilde{g}_{j}\right\}_{j=1}^{\infty}$, respectively, while $v$ and $\tilde{v}$ are solutions in Lemma 2.3 corresponding to $h(t)=w(0, t)$ and $h(t)=\tilde{w}(0, t)$, respectively. By applying (2.20) to $v-\tilde{v}$ and (3.6) to $w-\tilde{w}$, we have

$$
\begin{aligned}
& E\left(\sup _{s \in[0, t]}\|u(s)-\tilde{u}\|_{H^{1}(0, L)}^{2}\right)+E\left(\sup _{s \in[0, t]}\left\|u_{s}(s)-\tilde{u}_{s}(s)\right\|_{L^{2}(0, L)}^{2}\right) \\
& +E\left(\int_{0}^{t}\left|u_{x}(0, s)-\tilde{u}_{x}(0, s)\right|^{2} d s\right)+E\left(\int_{0}^{t}\left|u_{s}(0, s)-\tilde{u}_{s}(0, s)\right|^{2} d s\right) \\
& \leq M_{K} E\left(\sum_{j=1}^{\infty} \int_{0}^{t}\left\|g_{j}-\tilde{g}_{j}\right\|_{L^{2}(0, L)}^{2} d s\right), \quad \text { for all } t \in[0, T] .
\end{aligned}
$$

Let $u^{(0)}=u_{0}$, and for given $u^{(m)}, m \geq 0$, let $u^{(m+1)}$ be the solution of

$$
\begin{aligned}
& u_{t t}^{(m+1)}-u_{x x}^{(m+1)}+\alpha u_{t}^{(m+1)}=\sum_{j=1}^{\infty}\left(f_{j}+\sigma_{j} u^{(m)}\right) \frac{d B_{j}}{d t} \quad \text { for }(x, t) \in(0, L) \times(0, T), \\
& (4.5) \quad u_{x}^{(m+1)}(0, t)=-K u^{(m+1)}(0, t)^{-}, \quad u^{(m+1)}(L, t)=0 \quad \text { for } t \in(0, T), \\
& (4.6) \quad u^{(m+1)}(x, 0)=u_{0}(x), \quad u_{t}^{(m+1)}(x, 0)=u_{1}(x) \quad \text { for } x \in(0, L) .
\end{aligned}
$$

As in (4.3), we have

$$
\begin{aligned}
& E\left(\sup _{s \in[0, t]}\left\|u^{(m+1)}(s)-u^{(m)}(s)\right\|_{H^{1}(0, L)}^{2}\right)+E\left(\sup _{s \in[0, t]}\left\|u_{s}^{(m+1)}(s)-u_{s}^{(m)}(s)\right\|_{L^{2}(0, L)}^{2}\right) \\
& +E\left(\int_{0}^{t}\left|u_{x}^{(m+1)}(0, s)-u_{x}^{(m)}(0, s)\right|^{2} d s\right)+E\left(\int_{0}^{t}\left|u_{s}^{(m+1)}(0, s)-u_{s}^{(m)}(0, s)\right|^{2} d s\right) \\
& \leq M_{K} E\left(\int_{0}^{t}\left\|u^{(m)}(s)-u^{(m-1)}(s)\right\|_{L^{2}(0, L)}^{2} d s\right)
\end{aligned}
$$


for all $t \in[0, T]$, and all $m \geq 1$. By induction, we obtain

$$
\begin{aligned}
& E\left(\sup _{s \in[0, t]}\left\|u^{(m+1)}(s)-u^{(m)}(s)\right\|_{H^{1}(0, L)}^{2}\right)+E\left(\sup _{s \in[0, t]}\left\|u_{s}^{(m+1)}(s)-u_{s}^{(m)}(s)\right\|_{L^{2}(0, L)}^{2}\right) \\
& +E\left(\int_{0}^{t}\left|u_{x}^{(m+1)}(0, s)-u_{x}^{(m)}(0, s)\right|^{2} d s\right)+E\left(\int_{0}^{t}\left|u_{s}^{(m+1)}(0, s)-u_{s}^{(m)}(0, s)\right|^{2} d s\right) \\
& \leq M_{K}^{m} t^{m} / m ! \quad \text { for all } t \in[0, T], \text { and all } m \geq 1,
\end{aligned}
$$

where $M_{K}$ stands for a positive constant independent of $m$. Hence, $\left\{u^{(m)}\right\}_{m=1}^{\infty}$ is a Cauchy sequence in

$$
L^{2}\left(\Omega ; C\left([0, T] ; H^{1}(0, L)\right) \cap C^{1}\left([0, T] ; L^{2}(0, L)\right)\right)
$$

and each $u^{(m)}$ is progressively measurable. By the same argument as in the proof of Lemma 3.1, the limit $u$ is a solution of

$$
\begin{gathered}
u_{t t}-u_{x x}+\alpha u_{t}=\sum_{j=1}^{\infty}\left(f_{j}+\sigma_{j} u\right) \frac{d B_{j}}{d t} \quad \text { for }(x, t) \in(0, L) \times(0, T), \\
u_{x}(0, t)=-K u(0, t)^{-}, \quad u(L, t)=0 \quad \text { for } t \in(0, T), \\
u(x, 0)=u_{0}(x), \quad u_{t}(x, 0)=u_{1}(x) \quad \text { for } x \in(0, L)
\end{gathered}
$$

such that

$u \in L^{2}\left(\Omega ; C\left([0, T] ; H^{1}(0, L)\right)\right), u_{t} \in L^{2}\left(\Omega ; C\left([0, T] ; L^{2}(0, L)\right)\right)$, and $u_{t}(0, \cdot) \in$ $L^{2}\left(\Omega ; L^{2}(0, T)\right)$.

Taking this $u$ as a given function, let $w \in L^{2}\left(C\left([0, T] ; H^{1}(0, L)\right) \cap C^{1}([0, T]\right.$; $\left.\left.L^{2}(0, L)\right)\right)$ be a unique solution of

$$
\left\{\begin{array}{l}
w_{t t}-w_{x x}+\alpha w_{t}=\sum_{j=1}^{\infty}\left(f_{j}+\sigma_{j} u\right) \frac{d B_{j}}{d t} \quad \text { for }(x, t) \in(0, L) \times(0, T) \\
w_{x}(0, t)=0, \quad w(L, t)=0 \text { for } t \in(0, T) \\
w(x, 0)=u_{0}(x), \quad w_{t}(x, 0)=u_{1}(x) \quad \text { for } x \in(0, L)
\end{array}\right.
$$

By virtue of Lemma 2.3, we find that $v=u-w$ is a unique solution of (2.16) (2.18) for $h(t)=w(0, t)$ such that

$$
v \in L^{2}\left(\Omega ; C\left([0, T] ; H^{1}(0, L)\right) \cap C^{1}\left([0, T] ; L^{2}(0, L)\right)\right) .
$$


We now suppose $u_{0}(0) \geq 0$. It follows from (2.19) and (3.6) with $f \equiv 0, g_{j}=$ $f_{j}+\sigma_{j} u$ for $j \geq 1$, that

$$
\begin{aligned}
& E\left(\sup _{s \in[0, t]}\|u(s)\|_{H^{1}(0, L)}^{2}\right)+E\left(\sup _{s \in[0, t]}\left\|\partial_{s} u(s)\right\|_{L^{2}(0, L)}^{2}\right)+E\left(\int_{0}^{t}\left|\partial_{x} u(0, s)\right|^{2} d s\right) \\
& +E\left(\int_{0}^{t}\left|\partial_{s} u(0, s)\right|^{2} d s\right) \leq M E\left(\left\|u_{0}\right\|_{H^{1}(0, L)}^{2}\right)+M E\left(\left\|u_{1}\right\|_{L^{2}(0, L)}^{2}\right) \\
& \quad+M E\left(\int_{0}^{t}\|u(s)\|_{L^{2}(0, L)}^{2} d s\right)+M \sum_{j=1}^{\infty} E\left(\int_{0}^{t}\left\|f_{j}(s)\right\|_{L^{2}(0, L)}^{2} d s\right)
\end{aligned}
$$

for all $t \in[0, T]$, and thus, by the Gronwall inequality,

$$
\begin{aligned}
& E\left(\sup _{t \in[0, T]}\|u(t)\|_{H^{1}(0, L)}^{2}\right)+E\left(\sup _{t \in[0, T]}\left\|\partial_{t} u(t)\right\|_{L^{2}(0, L)}^{2}\right) \\
& +E\left(\int_{0}^{T}\left|\partial_{x} u(0, t)\right|^{2} d t\right)+E\left(\int_{0}^{T}\left|\partial_{t} u(0, t)\right|^{2} d t\right) \\
& \quad \leq M E\left(\left\|u_{0}\right\|_{H^{1}(0, L)}^{2}\right)+M E\left(\left\|u_{1}\right\|_{L^{2}(0, L)}^{2}\right)+M \sum_{j=1}^{\infty} E\left(\int_{0}^{T}\left\|f_{j}(t)\right\|_{L^{2}(0, L)}^{2} d t\right)
\end{aligned}
$$

where $M$ denotes positive constants independent of $K, u_{0}$ and $u_{1}$. Next we denote by $u_{K}$ the solution of (4.9) - (4.11) for each $K>0$. Since $L_{*}^{2}\left(\Omega ; L^{\infty}\left(0, T ; H^{n}(0, L)\right)\right)$ is the dual of $L^{2}\left(\Omega ; L^{1}\left(0, T ; H^{n}(0, L)\right)\right), n=0,1$, it follows from (4.14) that there is a sequence $\left\{u_{K_{m}}\right\}_{m=1}^{\infty}$ such that $K_{m} \uparrow \infty$ as $m \rightarrow \infty$, and

$$
\begin{gathered}
u_{K_{m}} \rightarrow u \quad \text { weak star in } L_{*}^{2}\left(\Omega ; L^{\infty}\left(0, T ; H^{1}(0, L)\right)\right), \\
\partial_{t} u_{K_{m}} \rightarrow \partial_{t} u \quad \text { weak star in } L_{*}^{2}\left(\Omega ; L^{\infty}\left(0, T ; L^{2}(0, L)\right)\right)
\end{gathered}
$$

as $m \rightarrow \infty$, for some $u$. Each $u_{K_{m}}$ satisfies

$$
\begin{gathered}
\int_{A} \int_{0}^{T}\left(\left\langle\partial_{t} u_{K_{m}}, \partial_{t} \psi\right\rangle-\left\langle\partial_{x} u_{K_{m}}, \partial_{x} \psi\right\rangle-\left\langle\alpha \partial_{t} u_{K_{m}}, \psi\right\rangle\right) d t d P \\
=\int_{A} \int_{0}^{T}\left\langle\partial_{t} \psi, \sum_{j=1}^{\infty} \int_{0}^{t}\left(f_{j}+\sigma_{j} u_{K_{m}}\right) d B_{j}\right\rangle d t d P
\end{gathered}
$$

for every $A \in \mathcal{F}$, and every $\psi \in H_{0}^{1}((0, L) \times(0, T))$. Here $\langle\cdot, \cdot\rangle$ denotes the inner product in $L^{2}(0, L)$. Since (4.15) and (4.16) imply that

$$
u_{K_{m}} \rightarrow u \quad \text { weakly in } L^{2}\left(\Omega ; L^{2}\left(0, T ; H^{1}(0, L)\right)\right) \text { as } m \rightarrow \infty
$$

and

$$
\partial_{t} u_{K_{m}} \rightarrow \partial_{t} u \quad \text { weakly in } L^{2}\left(\Omega ; L^{2}\left(0, T ; L^{2}(0, L)\right)\right) \text { as } m \rightarrow \infty,
$$

it follows from Lemma 1.1 that

$$
\begin{gathered}
\int_{A} \int_{0}^{T}\left(\left\langle\partial_{t} u, \partial_{t} \psi\right\rangle-\left\langle\partial_{x} u, \partial_{x} \psi\right\rangle-\left\langle\alpha \partial_{t} u, \psi\right\rangle\right) d t d P \\
=\int_{A} \int_{0}^{T}\left\langle\partial_{t} \psi, \sum_{j=1}^{\infty} \int_{0}^{t}\left(f_{j}+\sigma_{j} u\right) d B_{j}\right\rangle d t d P
\end{gathered}
$$


for every $A \in \mathcal{F}$, and every $\psi \in H_{0}^{1}((0, L) \times(0, T))$. Hence, there is $\tilde{\Omega} \subset \Omega$ such that $P(\Omega \backslash \tilde{\Omega})=0$ and for each $\omega \in \tilde{\Omega}$,

$$
\begin{gathered}
\int_{0}^{T}\left(\left\langle\partial_{t} u, \partial_{t} \psi\right\rangle-\left\langle\partial_{x} u, \partial_{x} \psi\right\rangle-\left\langle\alpha \partial_{t} u, \psi\right\rangle\right) d t \\
=\int_{0}^{T}\left\langle\partial_{t} \psi, \sum_{j=1}^{\infty} \int_{0}^{t}\left(f_{j}+\sigma_{j} u\right) d B_{j}\right\rangle d t
\end{gathered}
$$

for all $\psi$ in a countable dense subset of $H_{0}^{1}((0, L) \times(0, T))$. Consequently, (4.19) holds for every $\psi \in H_{0}^{1}((0, L) \times(0, T))$, for almost all $\omega$, because $u \in L_{*}^{2}(\Omega$; $\left.L^{\infty}\left(0, T ; H^{1}(0, L)\right)\right)$ and $u_{t} \in L_{*}^{2}\left(\Omega ; L^{\infty}\left(0, T ; L^{2}(0, L)\right)\right)$. Therefore, $u$ satisfies $(0.1)$ for almost all $\omega$.

Next we will show that $u$ also satisfies the boundary conditions. (4.15) implies that

$$
u_{K_{m}}(0, \cdot) \rightarrow u(0, \cdot) \quad \text { weak star in } L_{*}^{2}\left(\Omega ; L^{\infty}(0, T)\right)
$$

and

$$
u_{K_{m}}(L, \cdot) \rightarrow u(L, \cdot) \quad \text { weak star in } L_{*}^{2}\left(\Omega ; L^{\infty}(0, T)\right) .
$$

Thus, $u(L, \cdot) \equiv 0$, for almost all $\omega$. By the same argument as for (3.28), we find that

$$
\partial_{x x} u_{K_{m}} \rightarrow \partial_{x x} u \quad \text { weakly in } L^{2}\left(\Omega ; L^{2}\left(0, L ; H^{-1}(0, T)\right)\right)
$$

and hence,

$$
\partial_{x} u_{K_{m}}(0, \cdot) \rightarrow \partial_{x} u(0, \cdot) \quad \text { weakly in } L^{2}\left(\Omega ; H^{-1}(0, T)\right) .
$$

By virtue of (4.14) and (4.22),

$$
\partial_{x} u_{K_{m}}(0, \cdot) \rightarrow \partial_{x} u(0, \cdot) \quad \text { weakly in } L^{2}\left(\Omega ; L^{2}(0, T)\right) .
$$

Since

$$
\partial_{x} u_{K_{m}}(0, \cdot)=-K_{m} u_{K_{m}}(0, \cdot)^{-},
$$

we derive from (4.14) that

$$
u_{K_{m}}(0, \cdot)^{-} \rightarrow 0 \quad \text { strongly in } L^{2}\left(\Omega ; L^{2}(0, T)\right) .
$$

Choose any nonnegative $\phi \in L^{2}\left(\Omega ; L^{2}(0, T)\right)$. Then, it follows from (4.20) that

$$
\int_{\Omega} \int_{0}^{T}\left(u_{K_{m}}(0, t)^{+}-u_{K_{m}}(0, t)^{-}\right) \phi(t) d t d P \rightarrow \int_{\Omega} \int_{0}^{T} u(0, t) \phi(t) d t d P,
$$

which, combined with (4.25), yields

$$
\int_{\Omega} \int_{0}^{T} u(0, t) \phi(t) d t d P \geq 0
$$

Also, by (4.23) and (4.24), we have

$$
\int_{\Omega} \int_{0}^{T} \partial_{x} u(0, t) \phi(t) d t d P \leq 0
$$

Hence, we find that

$$
u(0, t)^{-}=0
$$


and

$$
\partial_{x} u(0, t) \leq 0
$$

for almost all $t \in[0, T]$, for almost all $\omega$. Next we show that $\left\{u_{K_{m}}(0, \cdot)\right\}_{m=1}^{\infty}$ is strongly convergent in $L^{2}\left(\Omega ; L^{2}(0, \delta)\right)$ for some $0<\delta<T$. Choose any $K_{m}<K_{n}$ and set

Then, we can write

$$
v_{1}=u_{K_{m}}, \quad v_{2}=u_{K_{n}} .
$$

$$
\partial_{x} v_{1}(0, t)-\partial_{x} v_{2}(0, t)=-K_{m}\left(v_{1}(0, t)^{-}-v_{2}(0, t)^{-}\right)+\left(K_{n}-K_{m}\right) v_{2}(0, t)^{-}
$$

and hence, by dropping all nonnegative terms,

$$
\begin{aligned}
& \left(\partial_{x} v_{1}(0, t)-\partial_{x} v_{2}(0, t)\right)\left(v_{1}(0, t)-v_{2}(0, t)\right) \\
& =-K_{m}\left(v_{1}(0, t)^{-}-v_{2}(0, t)^{-}\right)\left(v_{1}(0, t)^{+}-v_{2}(0, t)^{+}\right) \\
& +K_{m}\left(v_{1}(0, t)^{-}-v_{2}(0, t)^{-}\right)^{2}+\left(K_{n}-K_{m}\right) v_{2}(0, t)^{-}\left(v_{1}(0, t)^{+}-v_{2}(0, t)^{+}\right) \\
& -\left(K_{n}-K_{m}\right) v_{2}(0, t)^{-}\left(v_{1}(0, t)^{-}-v_{2}(0, t)^{-}\right) \\
& \geq-\left(K_{n}-K_{m}\right) v_{2}(0, t)^{-} v_{1}(0, t)^{-} .
\end{aligned}
$$

It follows from (4.14) and (4.24) that

$$
E\left(\int_{0}^{T}\left|v_{1}(0, t)^{-}\right|^{2} d t\right) \leq \frac{C}{K_{m}^{2}}
$$

and

$$
E\left(\int_{0}^{T}\left|v_{2}(0, t)^{-}\right|^{2} d t\right) \leq \frac{C}{K_{n}^{2}}
$$

where $C$ stands for the right-hand side of (4.14). Hence, we have

$$
\begin{aligned}
& E\left(\int_{0}^{T}\left(K_{n}-K_{m}\right) v_{2}(0, t)^{-} v_{1}(0, t)^{-} d t\right) \\
& \leq\left(\frac{K_{n}-K_{m}}{K_{m} K_{n}}\right) C .
\end{aligned}
$$

Applying Lemma 3.3 to $v=v_{1}-v_{2}$ with help from (4.29) and (4.32), there is some $0<\delta<T$ such that $\left\{u_{K_{m}}(0, \cdot)\right\}_{m=1}^{\infty}$ is a Cauchy sequence in $L^{2}\left(\Omega ; L^{2}(0, \delta)\right)$. Since

$$
\partial_{x} u_{K_{m}}(0, \cdot) \rightarrow \partial_{x} u(0, \cdot) \quad \text { weakly in } L^{2}\left(\Omega ; L^{2}(0, T)\right) \text { as } m \rightarrow \infty,
$$

we find that

$$
u_{K_{m}}(0, \cdot) \partial_{x} u_{K_{m}}(0, \cdot) \rightarrow u(0, \cdot) \partial_{x} u(0, \cdot) \quad \text { weakly in } L^{1}\left(\Omega ; L^{1}(0, \delta)\right) .
$$

But by (4.14) and (4.24) again, we see that as $m \rightarrow \infty$,

$$
u_{K_{m}}(0, \cdot) \partial_{x} u_{K_{m}}(0, \cdot)=K_{m}\left|u_{K_{m}}(0, \cdot)^{-}\right|^{2} \rightarrow 0 \quad \text { strongly in } L^{1}\left(\Omega ; L^{1}(0, T)\right) .
$$

Hence, it holds that

$$
u(0, t) \partial_{x} u(0, t)=0
$$

for almost all $t \in[0, \delta]$, for almost all $\omega$.

Therefore, $u$ is a solution of $(0.1)-(0.4)$ for $0<t<\delta$ such that

$$
u \in L_{*}^{2}\left(\Omega ; L^{\infty}\left(0, \delta ; H^{1}(0, L)\right)\right), \quad u_{t} \in L_{*}^{2}\left(\Omega ; L^{\infty}\left(0, \delta ; L^{2}(0, L)\right)\right) .
$$


Here we note that $\delta$ is independent of the initial conditions. Again by applying Lemma 2.1 to $u-w$ where $w$ is the solution of (4.12), we find that for almost all $\omega$,

$$
u \in C\left([0, \delta] ; H^{1}(0, L)\right), \quad u_{t} \in C\left([0, \delta] ; L^{2}(0, L)\right)
$$

by taking slightly smaller $\delta$. Since $C\left([0, \delta] ; H^{1}(0, L)\right)$ and $C\left([0, \delta] ; L^{2}(0, L)\right)$ are separable closed subspaces of $L^{\infty}\left(0, \delta ; H^{1}(0, L)\right)$ and $L^{\infty}\left(0, \delta ; L^{2}(0, L)\right)$, respectively, we have

$$
u \in L^{2}\left(\Omega ; C\left([0, \delta] ; H^{1}(0, L)\right)\right), \quad u_{t} \in L^{2}\left(\Omega ; C\left([0, \delta] ; L^{2}(0, L)\right)\right) .
$$

See [14, pp. $72-73]$. For the pathwise uniqueness of solution, we argue as follows. Let $v_{1}$ and $v_{2}$ be two solutions of $(0.1)-(0.4)$ such that

$$
v_{i} \in L^{2}\left(\Omega ; C\left([0, T] ; H^{1}(0, L)\right)\right), \quad \partial_{t} v_{i} \in L^{2}\left(\Omega ; C\left([0, T] ; L^{2}(0, L)\right)\right), \quad i=1,2,
$$

for some $T>0$. There is a subset $\tilde{\Omega} \subset \Omega$ such that $P(\Omega \backslash \tilde{\Omega})=0$, and for each $\omega \in \tilde{\Omega}, \quad(0.3)$ is satisfied by $v_{1}$ and $v_{2}$, for almost all $t \in[0, T]$. Fix any $\omega \in \tilde{\Omega}$ and $t$ where (0.3) is satisfied by $v_{1}$ and $v_{2}$. Set $v=v_{2}-v_{1}$. If $v(0, t)=0$, then $v(0, t) v_{x}(0, t)=0$ holds. If $v(0, t)>0$, then $v_{2}(0, t)>v_{1}(0, t) \geq 0$. Hence, $\partial_{x} v_{2}(0, t)=0$, and $v_{x}(0, t)=\partial_{x} v_{2}(0, t)-\partial_{x} v_{1}(0, t) \geq 0$. Thus, $v(0, t) v_{x}(0, t) \geq 0$.

By the same argument, if $v(0, t)<0$, then $v(0, t) v_{x}(0, t) \geq 0$ again holds. According to Remark 3.4, we conclude that $v \equiv 0$ on $[0, T]$. Finally, since $\delta$ in (4.34) is independent of the initial conditions, we can extend the time interval of existence by using the pathwise uniqueness of solution.

\section{§5. Proof of Theorem 1.5}

Throughout this section, we assume that $\sigma_{j} \equiv 0$, for all $j \geq 1$, and that all $f_{j}$ 's are deterministic and independent of time. The proof is based on the following result of $[10]$.

Suppose that $X(t, s ; z), 0 \leq s \leq t<\infty$ is a pathwise unique solution of a certain stochastic evolution equation such that $X(s, s ; z)=z$. We assume the following conditions.

[I] $X(\cdot, s ; z)$ is a $\Xi$-valued continuous process adapted to $\left\{\mathcal{F}_{t}\right\}_{t \geq s}$ for each $z \in \Xi$ and $s \geq 0$, where $\Xi$ is a separable Banach space.

We define a function

$$
\mathcal{P}(s, z ; t, \Gamma)=P(X(t, s ; z) \in \Gamma), \quad \text { for each } \Gamma \in \mathcal{B}(\Xi), 0 \leq s \leq t<\infty, z \in \Xi
$$

where $\mathcal{B}(\Xi)$ is the Borel $\sigma$-algebra of $\Xi$.

[II] $\mathcal{P}(\cdot, \cdot ; \cdot, \cdot)$ is a time-homogeneous transition probability function. In other words, it satisfies the following conditions.

(i) $\mathcal{P}(s, z ; t, \cdot)$ is a probability measure over $(\Xi, \mathcal{B}(\Xi))$ for all $z \in \Xi$, and $0 \leq s<$ $t<\infty$.

(ii) $\mathcal{P}(s, \cdot ; t, \Gamma)$ is $\mathcal{B}(\Xi)$-measurable for all $0 \leq s<t<\infty$ and $\Gamma \in \mathcal{B}(\Xi)$.

(iii) For all $0 \leq s<t<\xi<\infty$ and $\Gamma \in \mathcal{B}(\Xi)$,

$$
\mathcal{P}(s, z ; \xi, \Gamma)=\int_{\Xi} \mathcal{P}(s, z ; t, d y) \mathcal{P}(t, y ; \xi, \Gamma) .
$$

(iv) $\mathcal{P}(s, \cdot ; t, \cdot)=\mathcal{P}(s+h, \cdot ; t+h, \cdot)$ for all $0 \leq s<t<\infty$ and $h>0$.

[III] If $\|z\|_{\Xi} \leq M$, then

$$
E\left(\|X(t, 0 ; z)\|_{\Xi}\right) \leq C_{M}, \quad \text { for all } t \geq 0,
$$

for some positive constant $C_{M}$. 
[IV] There is a separable Banach space $\Upsilon$ such that $\Xi \subset \Upsilon$, the imbedding $\Xi \rightarrow \Upsilon$ is continuous, and each closed ball of finite radius in $\Xi$ is a compact subset of $\Upsilon$. Furthermore, for each bounded continuous function $\psi$ on $\Xi$, there is a sequence of continuous functions $\left\{\psi_{k}\right\}_{k=1}^{\infty}$ on $\Upsilon$ such that $\psi_{k}$ is bounded uniformly in $k$ and

$$
\lim _{k \rightarrow \infty} \psi_{k}(y)=\psi(y), \quad \text { for each } y \in \Xi .
$$

[V] For each fixed $0 \leq t<\infty$, and each fixed closed ball $S$ of finite radius in $\Xi$, if $\left\{z_{n}\right\}_{n=1}^{\infty}$ is a sequence in $S$ such that

$$
z_{n} \rightarrow z \quad \text { in } \Upsilon
$$

then

$$
E\left(\phi\left(X\left(t, 0 ; z_{n}\right)\right)\right) \rightarrow E(\phi(X(t, 0 ; z))),
$$

for every bounded continuous function $\phi$ on $\Upsilon$.

Theorem ([10]). Under the assumptions $[\mathrm{I}]-[\mathrm{V}]$, there is an invariant measure for the above process $X(\cdot)$. In other words, there is a probability measure $\mu$ on $\Xi$ such that

$$
\int_{\Xi} E(\psi(X(t, 0 ; z))) \mu(d z)=\int_{\Xi} \psi(z) \mu(d z)
$$

for all $t \geq 0$, and every bounded continuous function $\psi$ on $\Xi$.

For the proof of Theorem 1.5, it is enough to establish the above assumptions $[\mathrm{I}]-[\mathrm{V}]$.

We will first prove pathwise convergence of approximate solutions, where the assumption $\sigma_{j} \equiv 0, j \geq 1$, is essentially used.

Lemma 5.1. Let $u_{K}$ be the solution of (4.9) - (4.11) with $\sigma_{j} \equiv 0, j \geq 1$. Then, for each sequence $\left\{u_{K_{m}}\right\}_{m=1}^{\infty}$, it holds that for almost all $\omega$,

$$
\begin{gathered}
u_{K_{m}} \rightarrow u \quad \text { weak star in } L^{\infty}\left(0, T ; H^{1}(0, L)\right), \\
\partial_{t} u_{K_{m}} \rightarrow \partial_{t} u \quad \text { weak star in } L^{\infty}\left(0, T ; L^{2}(0, L)\right)
\end{gathered}
$$

as $K_{m} \uparrow \infty$, for each $T>0$, where $u$ is the solution in Theorem 1.4.

Proof. Choose any $T>0$, and let $w$ be the solution of (4.12) with $\sigma_{j} \equiv 0, j \geq 1$. Then, there is some $\tilde{\Omega} \subset \Omega$ such that $P(\Omega \backslash \tilde{\Omega})=0$, and for each $\omega \in \tilde{\Omega}$,

$$
\begin{gathered}
w \in C\left([0, T] ; H^{1}(0, L)\right) \cap C^{1}\left([0, T] ; L^{2}(0, L)\right), \quad w(0, \cdot) \in H^{1}(0, T), \\
\Phi=\sum_{j=1}^{\infty} f_{j} B_{j}(\cdot) \in C\left([0, T] ; L^{2}(0, L)\right)
\end{gathered}
$$

and $w$ satisfies (4.12).

Let $v_{K}$ be the solution of $(2.16)-(2.18)$ with $h(t)=w(0, t)$. Then, according to the construction of the solution $u_{K}$ of (4.9) - (4.11) with $\sigma_{j} \equiv 0, j \geq 1$,

$$
u_{K}=w+v_{K}, \quad \text { for each } \omega \in \tilde{\Omega} .
$$

It follows from (2.19) that

$$
\begin{aligned}
& \left\|\partial_{t} v_{K}(t)\right\|_{L^{2}(0, L)}^{2}+\left\|v_{K}(t)\right\|_{H^{1}(0, L)}^{2}+\int_{0}^{t}\left|\partial_{x} v_{K}(0, s)\right|^{2} d s \\
& \leq M \int_{0}^{t}\left|w_{s}(0, s)\right|^{2} d s
\end{aligned}
$$


for all $t \in[0, T]$, for each $\omega \in \tilde{\Omega}$. Here $M$ is a constant independent of $K$ and $\omega$. Now fix any $\omega \in \tilde{\Omega}$. By (5.3), there is a sequence $\left\{v_{K_{m}}\right\}_{m=1}^{\infty}$ such that

$$
\begin{gathered}
v_{K_{m}} \rightarrow v_{\omega} \quad \text { weak star in } L^{\infty}\left(0, T ; H^{1}(0, L)\right), \\
\partial_{t} v_{K_{m}} \rightarrow \partial_{t} v_{\omega} \quad \text { weak star in } L^{\infty}\left(0, T ; L^{2}(0, L)\right)
\end{gathered}
$$

for some function $v_{\omega}$. This $v_{\omega}$ must satisfy (2.16), (2.18) and

$$
\left\{\begin{array}{l}
v_{\omega}(0, t)+w(0, t) \geq 0 \\
\partial_{x} v_{\omega}(0, t) \leq 0 \\
\partial_{x} v_{\omega}(0, t)\left(v_{\omega}(0, t)+w(0, t)\right)=0 \\
v_{\omega}(L, t)=0
\end{array}\right.
$$

for almost all $t \in[0, T]$. Here we note that (5.4) and (5.5) imply that for any $\eta>0$,

$$
v_{K_{m}} \rightarrow v_{\omega} \quad \text { strongly in } C\left([0, T] ; H^{1-\eta}(0, L)\right)
$$

and hence,

$$
v_{K_{m}}(0, \cdot) \rightarrow v_{\omega}(0, \cdot) \quad \text { strongly in } C([0, T]) .
$$

For (5.7), see Lemma 1.7 of [9]. Meanwhile, by (5.3),

$\partial_{x} v_{K_{m}}(0, \cdot)\left(v_{K_{m}}(0, \cdot)+w(0, \cdot)\right)=K_{m}\left|\left(v_{K_{m}}(0, \cdot)+w(0, \cdot)\right)^{-}\right|^{2} \rightarrow 0 \quad$ in $L^{1}(0, T)$, which yields the third property in (5.6). Next it follows from Lemma 2.1 that

$$
v_{\omega} \in C\left([0, T) ; H^{1}(0, L)\right) \cap C^{1}\left([0, T) ; L^{2}(0, L)\right) .
$$

Suppose $\tilde{v}_{\omega}$ is a limit of another sequence. Then, by the same argument as in Section $4, v_{\omega}-\tilde{v}_{\omega}$ satisfies the boundary condition

$$
\left(v_{\omega}(0, t)-\tilde{v}_{\omega}(0, t)\right)\left(\partial_{x} v_{\omega}(0, t)-\partial_{x} \tilde{v}_{\omega}(0, t)\right) \geq 0
$$

for almost all $t \in[0, T]$, for each $\omega \in \tilde{\Omega}$. Again by Remark 3.4, we conclude that $v_{\omega} \equiv \tilde{v}_{\omega}$. Hence, every sequence converges to the same limit. We now fix any sequence $\left\{v_{K_{m}}\right\}_{m=1}^{\infty}$. For each $\omega \in \tilde{\Omega}$, we define $v_{\omega}$ as the limit of this sequence. Since $C\left([0, T-\epsilon] ; H^{1}(0, L)\right)$ is a separable closed subspace of $L^{\infty}\left(0, T-\epsilon ; H^{1}(0, L)\right)$, for each $0<\epsilon<T$, it follows from (5.4) and (5.8) that $v=v_{\omega}$ is $C\left([0, T-\epsilon] ; H^{1}(0, L)\right)$ valued strongly measurable over $\left(\Omega, \mathcal{F}_{T-\epsilon}\right)$, which implies that $v$ is also progressively measurable. Similarly, $\partial_{t} v$ is $C\left([0, T-\epsilon] ; L^{2}(0, L)\right)$-valued strongly measurable over $\left(\Omega, \mathcal{F}_{T-\epsilon}\right)$. By virtue of $(5.4),(5.5)$ and Fatou's lemma, it holds that for each $0<\epsilon<T$,

$$
\begin{aligned}
& E\left(\|v+w\|_{C\left([0, T-\epsilon] ; H^{1}(0, L)\right)}^{2}+\left\|v_{t}+w_{t}\right\|_{C\left([0, T-\epsilon] ; L^{2}(0, L)\right)}^{2}\right) \\
& \leq E\left(\underset{m \rightarrow \infty}{\underline{\lim }}\left(\left\|v_{K_{m}}+w\right\|_{L^{\infty}\left(0, T ; H^{1}(0, L)\right)}^{2}+\left\|\partial_{t} v_{K_{m}}+w_{t}\right\|_{L^{\infty}\left(0, T ; H^{1}(0, L)\right)}^{2}\right)\right) \\
& \leq \underset{m \rightarrow \infty}{\lim } E\left(\left\|v_{K_{m}}+w\right\|_{L^{\infty}\left(0, T ; H^{1}(0, L)\right)}^{2}+\left\|\partial_{t} v_{K_{m}}+w_{t}\right\|_{L^{\infty}\left(0, T ; H^{1}(0, L)\right)}^{2}\right),
\end{aligned}
$$

which, together with (5.3), yields

$$
v+w \in L^{2}\left(\Omega ; C\left([0, T-\epsilon] ; H^{1}(0, L)\right)\right), \quad v_{t}+w_{t} \in L^{2}\left(\Omega ; C\left([0, T-\epsilon] ; L^{2}(0, L)\right)\right) .
$$


Obviously, $v+w$ is a solution of $(0.1)-(0.4)$ on the interval $[0, T-\epsilon]$, for each $0<\epsilon<T$. By the pathwise uniqueness of the solution, this coincides with the solution in Theorem 1.4, and the proof of Lemma 5.1 is complete.

We next establish the following estimate.

Lemma 5.2. Let $u$ be the solution in Theorem 1.4 under the conditions of Theorem 1.5. It holds that

$$
E\left(\|u(t)\|_{H^{1}(0, L)}^{2}+\left\|u_{t}(t)\right\|_{L^{2}(0, L)}^{2}\right) \leq M
$$

for all $t \geq 0$, for some constant $M>0$.

Proof. Fix any $T>0$ and $K=k$. Let $w$ be the solution of (4.12) with $\sigma_{j} \equiv$ $0, j \geq 1$, and let $v_{k}$ be the solution in Lemma 2.3 with $h=w(0, \cdot)$. We write $u_{k}=v_{k}+w$. As above, let us define

$$
\begin{aligned}
u_{0, \nu} & =\sum_{k=1}^{\nu}\left\langle u_{0}, e_{k}\right\rangle e_{k}, & u_{1, \nu} & =\sum_{k=1}^{\nu}\left\langle u_{1}, e_{k}\right\rangle e_{k}, \\
f_{j, \nu} & =\sum_{k=1}^{\nu}\left\langle f_{j}, e_{k}\right\rangle e_{k}, & \Phi_{\nu} & =\sum_{j=1}^{\infty} f_{j, \nu} B_{j}(\cdot) .
\end{aligned}
$$

Let $w_{\nu}$ be the solution in Lemma 3.2 with $f_{\nu}+\partial_{t} \Psi_{\nu}$ replaced by $\partial_{t} \Phi_{\nu}$. Then, as $\nu \rightarrow \infty$,

$$
\begin{gathered}
w_{\nu} \rightarrow w \quad \text { in } L^{2}\left(\Omega ; C\left([0, T] ; H^{1}(0, L)\right) \cap C^{1}\left([0, T] ; L^{2}(0, L)\right)\right), \\
w_{\nu}(0, \cdot) \rightarrow w(0, \cdot) \quad \text { in } L^{2}\left(\Omega ; H^{1}(0, T)\right) .
\end{gathered}
$$

After extending $-k\left(v_{k}(0, t)+w(0, t)\right)^{-}$to be zero for $t \notin[0, T]$, we define

$$
q_{k, \nu}(t)=-\int_{-\infty}^{\infty} k\left(v_{k}(0, t-s)+w(0, t-s)\right)^{-} \rho_{\nu}(s) d s
$$

where $\rho_{\nu}(t)=\nu \rho(\nu t), \rho \in C_{0}^{\infty}((0,1))$ such that $\|\rho\|_{L^{1}(0,1)}=1$.

Then, $q_{k, \nu} \in C_{0}^{\infty}(R)$ is adapted to $\left\{\mathcal{F}_{t}\right\}$ and, as $\nu \rightarrow \infty$,

$$
q_{k, \nu} \rightarrow-k\left(v_{k}(0, \cdot)+w(0, \cdot)\right)^{-} \quad \text { in } L^{2}\left(\Omega ; L^{2}(0, T)\right) .
$$

Let $v_{k, \nu}$ be the solution in Lemma 2.2 with $q=q_{k, \nu}$ and $f \equiv 0$, satisfying the additional regularity (2.13). Then, as $\nu \rightarrow \infty$,

$$
v_{k, \nu} \rightarrow v_{k} \quad \text { in } L^{2}\left(\Omega ; C\left([0, T] ; H^{1}(0, L)\right) \cap C^{1}\left([0, T] ; L^{2}(0, L)\right)\right)
$$

and

$$
\partial_{t} v_{k, \nu}(0, \cdot) \rightarrow \partial_{t} v_{k}(0, \cdot) \quad \text { in } L^{2}\left(\Omega ; L^{2}(0, L)\right)
$$

We may write

$$
d\left(\partial_{t} w_{\nu}+\partial_{t} v_{k, \nu}\right)=\left(\partial_{x x} w_{\nu}+\partial_{x x} v_{k, \nu}\right) d t-\alpha\left(\partial_{t} w_{\nu}+\partial_{t} v_{k, \nu}\right) d t+d \Phi_{\nu}
$$

and

$$
d\left(w_{\nu}+v_{k, \nu}\right)=\left(\partial_{t} w_{\nu}+\partial_{t} v_{k, \nu}\right) d t
$$

By applying Ito's rule to the functionals

$$
\left\langle\partial_{t} w_{\nu}+\partial_{t} v_{k, \nu}, \partial_{t} w_{\nu}+\partial_{t} v_{k, \nu}\right\rangle
$$


and

$$
\left\langle\partial_{t} w_{\nu}+\partial_{t} v_{k, \nu}, w_{\nu}+v_{k, \nu}\right\rangle
$$

we have for each $\nu \geq 1$,

$$
\begin{aligned}
& \left\|\partial_{t} w_{\nu}(t)+\partial_{t} v_{k, \nu}(t)\right\|_{L^{2}(0, L)}^{2}+\left\|\partial_{x} w_{\nu}(t)+\partial_{x} v_{k, \nu}(t)\right\|_{L^{2}(0, L)}^{2} \\
& =\left\|\partial_{x} u_{0, \nu}\right\|_{L^{2}(0, L)}^{2}+\left\|u_{1, \nu}\right\|_{L^{2}(0, L)}^{2} \\
& -2 \int_{0}^{t} q_{k, \nu}(s)\left(\partial_{s} v_{k, \nu}(0, s)+\partial_{s} w_{\nu}(0, s)\right) d s \\
& -2 \alpha \int_{0}^{t}\left\|\partial_{s} w_{\nu}(s)+\partial_{s} v_{k, \nu}(s)\right\|_{L^{2}(0, L)}^{2} d s \\
& +2 \sum_{j=1}^{\infty} \int_{0}^{t}\left\langle\partial_{s} w_{\nu}(s)+\partial_{s} v_{k, \nu}(s), f_{j, \nu}\right\rangle d B_{j}(s)+\sum_{j=1}^{\infty} t\left\|f_{j, \nu}\right\|_{L^{2}(0, L)}^{2}
\end{aligned}
$$

and

$$
\begin{aligned}
& \epsilon\left\langle\partial_{t} w_{\nu}(t)+\partial_{t} v_{k, \nu}(t), w_{\nu}(t)+v_{k, \nu}(t)\right\rangle-\epsilon\left\langle u_{0, \nu}, u_{1, \nu}\right\rangle \\
& +\frac{\epsilon \alpha}{2}\left\|w_{\nu}(t)+v_{k, \nu}(t)\right\|_{L^{2}(0, L)}^{2}-\frac{\epsilon \alpha}{2}\left\|u_{0, \nu}\right\|_{L^{2}(0, L)}^{2} \\
& =-\epsilon \int_{0}^{t} q_{k, \nu}(s)\left(v_{k, \nu}(0, s)+w_{\nu}(0, s)\right) d s \\
& -\epsilon \int_{0}^{t}\left\|\partial_{x} w_{\nu}(s)+\partial_{x} v_{k, \nu}(s)\right\|_{L^{2}(0, L)}^{2} d s \\
& +\epsilon \int_{0}^{t}\left\|\partial_{s} w_{\nu}(s)+\partial_{s} v_{k, \nu}(s)\right\|_{L^{2}(0, L)}^{2} d s \\
& +\epsilon \sum_{j=1}^{\infty} \int_{0}^{t}\left\langle w_{\nu}(s)+v_{k, \nu}(s), f_{j, \nu}\right\rangle d B_{j}(s)
\end{aligned}
$$

for all $t \in[0, T]$, for almost all $\omega$. Here $\epsilon$ is a positive constant, which will be determined later. By means of (5.9) - (5.13), we can extract a subsequence $\left\{w_{\nu_{m}}+\right.$ $\left.v_{k, \nu_{m}}\right\}_{m=1}^{\infty}$ for pathwise convergence and pass $\nu_{m} \rightarrow \infty$ in (5.14) and (5.15) to arrive at

$$
\begin{aligned}
& \left\|\partial_{t} u_{k}(t)\right\|_{L^{2}(0, L)}^{2}+\left\|\partial_{x} u_{k}(t)\right\|_{L^{2}(0, L)}^{2}=\left\|\partial_{x} u_{0}\right\|_{L^{2}(0, L)}^{2}+\left\|u_{1}\right\|_{L^{2}(0, L)}^{2} \\
& +2 \int_{0}^{t} k u_{k}(0, s)^{-} \partial_{s} u_{k}(0, s) d s-2 \alpha \int_{0}^{t}\left\|\partial_{s} u_{k}(s)\right\|_{L^{2}(0, L)}^{2} d s \\
& +2 \sum_{j=1}^{\infty} \int_{0}^{t}\left\langle\partial_{s} u_{k}, f_{j}\right\rangle d B_{j}(s)+\sum_{j=1}^{\infty} t\left\|f_{j}\right\|_{L^{2}(0, L)}^{2}
\end{aligned}
$$

and

$$
\begin{aligned}
& \epsilon\left\langle\partial_{t} u_{k}(t), u_{k}(t)\right\rangle-\epsilon\left\langle u_{0}, u_{1}\right\rangle+\frac{\epsilon \alpha}{2}\left\|u_{k}(t)\right\|_{L^{2}(0, L)}^{2}-\frac{\epsilon \alpha}{2}\left\|u_{0}\right\|_{L^{2}(0, L)}^{2} \\
& =\epsilon \int_{0}^{t} k u_{k}(0, s)^{-} u_{k}(0, s) d s-\epsilon \int_{0}^{t}\left\|\partial_{x} u_{k}(s)\right\|_{L^{2}(0, L)}^{2} d s \\
& +\epsilon \int_{0}^{t}\left\|\partial_{s} u_{k}(s)\right\|_{L^{2}(0, L)}^{2} d s+\epsilon \sum_{j=1}^{\infty} \int_{0}^{t}\left\langle u_{k}(s), f_{j}\right\rangle d B_{j}(s)
\end{aligned}
$$


for all $t \in[0, T]$, for almost all $\omega \in \Omega$. Since $T>0$ could be chosen arbitrarily, (5.16) and (5.17) are valid for all $t \geq 0$, for almost all $\omega$.

Next, by means of $(1.1)$ and $u_{0}(0) \geq 0$, we find that

$$
\int_{0}^{t} k u_{k}(0, s)^{-} \partial_{s} u_{k}(0, s) d s=-\frac{k}{2}\left|u_{k}(0, t)^{-}\right|^{2}
$$

Let us set

$$
Q_{k}(t)=\left\|\partial_{t} u_{k}(t)\right\|_{L^{2}(0, L)}^{2}+\left\|\partial_{x} u_{k}(t)\right\|_{L^{2}(0, L)}^{2}+\epsilon\left\langle\partial_{t} u_{k}(t), u_{k}(t)\right\rangle+\frac{\epsilon \alpha}{2}\left\|u_{k}(t)\right\|_{L^{2}(0, L)}^{2}
$$

and choose sufficiently small $0<\epsilon<\alpha$ such that

$c_{1}\left(\left\|\partial_{t} u_{k}(t)\right\|_{L^{2}(0, L)}^{2}+\left\|u_{k}(t)\right\|_{H^{1}(0, L)}^{2}\right) \leq Q_{k}(t) \leq c_{2}\left(\left\|\partial_{t} u_{k}(t)\right\|_{L^{2}(0, L)}^{2}+\left\|u_{k}(t)\right\|_{H^{1}(0, L)}^{2}\right)$

for some positive constants $c_{1}, c_{2}$ independent of $u_{k}(t)$. We now set

$$
\mathcal{R}_{k}(t)=Q_{k}(t)+k\left|u_{k}(0, t)^{-}\right|^{2} .
$$

It follows that for any $0 \leq t_{1}<t_{2}$,

$$
\begin{aligned}
& E\left(\mathcal{R}_{k}\left(t_{2}\right)\right)-E\left(\mathcal{R}_{k}\left(t_{1}\right)\right)=-(2 \alpha-\epsilon) \int_{t_{1}}^{t_{2}} E\left(\left\|\partial_{t} u_{k}(t)\right\|_{L^{2}(0, L)}^{2}\right) d t \\
& -\epsilon \int_{t_{1}}^{t_{2}} E\left(k\left|u_{k}(0, t)^{-}\right|^{2}\right) d t-\epsilon \int_{t_{1}}^{t_{2}} E\left(\left\|\partial_{x} u_{k}(t)\right\|_{L^{2}(0, L)}^{2}\right) d t \\
& +\sum_{j=1}^{\infty}\left(t_{2}-t_{1}\right)\left\|f_{j}\right\|_{L^{2}(0, L)}^{2} .
\end{aligned}
$$

Hence, there is some positive constant $c$ independent of $k$ such that

$$
\frac{d}{d t} E\left(\mathcal{R}_{k}(t)\right) \leq-c E\left(\mathcal{R}_{k}(t)\right)+\sum_{j=1}^{\infty}\left\|f_{j}\right\|_{L^{2}(0, L)}^{2}
$$

for all $t>0$, which implies that

$$
E\left(\mathcal{R}_{k}(t)\right) \leq M, \quad \text { for all } k \geq 1, \text { and all } t \geq 0 .
$$

Meanwhile, according to Lemma 5.1, there is $\tilde{\Omega} \subset \Omega$ such that $P(\Omega \backslash \tilde{\Omega})=0$ and for each $\omega \in \tilde{\Omega}$,

$$
\begin{gathered}
u_{k} \rightarrow u \quad \text { weak star in } L^{\infty}\left(0, T ; H^{1}(0, L)\right) \text {, as } k \rightarrow \infty, \\
\partial_{t} u_{k} \rightarrow u_{t} \quad \text { weak star in } L^{\infty}\left(0, T ; L^{2}(0, L)\right) \text {, as } k \rightarrow \infty, \\
\Phi \in C\left([0, T] ; L^{2}(0, L)\right)
\end{gathered}
$$

and

$u$ and all $u_{k}$ 's satisfy $(0.1)$ in the sense of distributions over $(0, L) \times(0, T)$, for all $T>0$. It follows that for each $\omega \in \tilde{\Omega}$,

$$
\partial_{t}\left(\partial_{t} u_{k}-\Phi\right) \rightarrow \partial_{t}\left(u_{t}-\Phi\right) \quad \text { weak star in } L^{\infty}\left(0, T ; H^{-1}(0, L)\right),
$$

as $k \rightarrow \infty$. Thus, for each $\omega \in \tilde{\Omega}$, it holds that

$$
u_{k} \rightarrow u \quad \text { in } C\left([0, T] ; H^{1-\eta}(0, L)\right)
$$

and

$$
\partial_{t} u_{k} \rightarrow u_{t} \quad \text { in } C\left([0, T] ; H^{-\eta}(0, L)\right)
$$


for any $\eta>0$, for all $T>0$. See Lemma 1.7 of [9]. Consequently, for all $t \geq 0$, and all $\omega \in \tilde{\Omega}$,

$$
u_{k}(t) \rightarrow u(t) \quad \text { in } H^{1-\eta}(0, L)
$$

and

$$
\partial_{t} u_{k}(t) \rightarrow u_{t}(t) \quad \text { in } H^{-\eta}(0, L),
$$

as $k \rightarrow \infty$. Now fix any $t \geq 0$. Define

$$
\mathcal{S}_{k}(t)=\left\|u_{k}(t)\right\|_{H^{1}(0, L)}^{2}+\left\|\partial_{t} u_{k}(t)\right\|_{L^{2}(0, L)}^{2} .
$$

By Fatou's lemma, (5.20) yields

$$
E\left(\varliminf_{k \rightarrow \infty} \mathcal{S}_{k}(t)\right) \leq M
$$

where $M$ is a positive constant independent of $t$. This implies that there is some $\Omega_{t} \subset \Omega$ such that $P\left(\Omega \backslash \Omega_{t}\right)=0$ and, for each $\omega \in \Omega_{t}$,

$$
\varliminf_{k \rightarrow \infty} \mathcal{S}_{k}(t)<\infty,
$$

which, combined with (5.28) and (5.29), implies that for each fixed $\omega \in \tilde{\Omega} \cap \Omega_{t}$, there is a subsequence $\left\{u_{k_{m}}\right\}_{m=1}^{\infty}$ such that

$$
\begin{gathered}
\lim _{m \rightarrow \infty} \mathcal{S}_{k_{m}}(t)=\varliminf_{k \rightarrow \infty} \mathcal{S}_{k}(t)<\infty, \\
u_{k_{m}}(t) \rightarrow u(t) \quad \text { weakly in } H^{1}(0, L)
\end{gathered}
$$

and

$$
\partial_{t} u_{k_{m}}(t) \rightarrow u_{t}(t) \quad \text { weakly in } L^{2}(0, L)
$$

Consequently,

$$
\|u(t)\|_{H^{1}(0, L)}^{2}+\left\|u_{t}(t)\right\|_{L^{2}(0, L)}^{2} \leq \varliminf_{k \rightarrow \infty} \mathcal{S}_{k}(t) .
$$

This is true for each $\omega \in \Omega_{t} \cap \tilde{\Omega}$. Hence, we have

$$
E\left(\|u(t)\|_{H^{1}(0, L)}^{2}+\left\|u_{t}(t)\right\|_{L^{2}(0, L)}^{2}\right) \leq M
$$

for all $t \geq 0$. This proves the lemma.

Next we define

$$
\mathcal{Y}=\left\{z=\left(z_{1}, z_{2}\right) \quad \mid \quad z_{1} \in H^{1}(0, L), z_{1}(0) \geq 0, z_{1}(L)=0, \quad z_{2} \in L^{2}(0, L)\right\} .
$$

$\mathcal{Y}$ is a closed subset of $H^{1}(0, L) \times L^{2}(0, L)$, and thus, $\mathcal{B}(\mathcal{Y}) \subset \mathcal{B}\left(H^{1}(0, L) \times\right.$ $\left.L^{2}(0, L)\right)$. For each $z \in \mathcal{Y}$, we write

$$
X(t ; s, z)=\left(u(t), u_{t}(t)\right), \quad \text { for } t \geq s \geq 0
$$

where $u$ is the solution of $(0.1)-(0.4)$ with the initial condition $\left(u(s), u_{t}(s)\right)=z$. We then write

$$
\mathcal{P}(s, z ; t, \Gamma)=P\{X(t ; s, z) \in \Gamma\}
$$

for each $\Gamma \in \mathcal{B}\left(H^{1}(0, L) \times L^{2}(0, L)\right)$ and $t \geq s \geq 0$. 
Lemma 5.3. For each $t>s \geq 0$, and $z \in \mathcal{Y}, \mathcal{P}(s, z ; t, \cdot)$ is a probability measure over $\left(H^{1}(0, L) \times L^{2}(0, L), \mathcal{B}\left(H^{1}(0, L) \times L^{2}(0, L)\right)\right)$ supported on $\mathcal{Y}$. For each $t>s \geq 0$ and $\Gamma \in \mathcal{B}\left(H^{1}(0, L) \times L^{2}(0, L)\right), \mathcal{P}(s, \cdot ; t, \Gamma)$ is $\mathcal{B}\left(H^{1}(0, L) \times L^{2}(0, L)\right)$ measurable.

Proof. The first assertion is obvious from Theorem 1.4. We will prove the second assertion. Choose any arbitrary sequence $\left\{z_{n}\right\}_{n=1}^{\infty}$ in $\mathcal{Y}$ such that $z_{n} \rightarrow z$ in $H^{1}(0, L) \times L^{2}(0, L)$, as $n \rightarrow \infty$. Let $w_{n}$ be the solution of (4.12) with $\sigma_{j} \equiv 0, j \geq 1$, and the initial condition $\left(w_{n}(s), \partial_{t} w_{n}(s)\right)=z_{n}$ for each $n \geq 1$. Let $v_{k, n}$ be the solution in Lemma 2.3 with $K=k, h=w_{n}(0, \cdot)$ and the initial condition $v_{k, n}(\cdot, s)=0, \partial_{t} v_{k, n}(\cdot, s)=0$. We write

$$
X_{k}\left(\cdot, s ; z_{n}\right)=\left(w_{n}+v_{k, n}, \partial_{t} w_{n}+\partial_{t} v_{k, n}\right) .
$$

Then, for each $t \geq s \geq 0$,

$$
X_{k}\left(t, s ; z_{n}\right) \rightarrow X_{k}(t, s ; z) \quad \text { in } L^{2}\left(\Omega ; H^{1}(0, L) \times L^{2}(0, L)\right)
$$

as $n \rightarrow \infty$. We define a function space $H^{*}(0, L)$ by

$$
H^{*}(0, L)=\left\{\sum_{k=1}^{\infty} d_{k} e_{k} \mid \sum_{k=1}^{\infty} \frac{\left|d_{k}\right|^{2}}{\lambda_{k}}<\infty\right\}
$$

where the $\lambda_{k}$ 's and $e_{k}$ 's are the same as in (3.7).

Let $\psi$ be a bounded continuous function on $L^{2}(0, L) \times H^{*}(0, L)$. Then, it is also a bounded continuous function on $H^{1}(0, L) \times L^{2}(0, L)$. It follows from (5.32) that there is a subsequence $\left\{X_{k}\left(t, s ; z_{n_{m}}\right)\right\}_{m=1}^{\infty}$ that converges to $X_{k}(t, s ; z)$ in $H^{1}(0, L) \times L^{2}(0, L)$, for almost all $\omega$. This implies that

$$
\int_{\Omega} \psi\left(X_{k}\left(t, s ; z_{n}\right)\right) d P \rightarrow \int_{\Omega} \psi\left(X_{k}(t, s ; z)\right) d P
$$

as $n \rightarrow \infty$, and hence,

$$
\int_{\Omega} \psi\left(X_{k}(t, s ; z)\right) d P \quad \text { is continuous in } z \in \mathcal{Y} .
$$

Meanwhile, it follows from (5.26) and (5.27) that for almost all $\omega \in \Omega$,

$$
X_{k}(t, s ; z) \rightarrow X(t, s ; z) \quad \text { in } L^{2}(0, L) \times H^{*}(0, L)
$$

as $k \rightarrow \infty$, because $H^{-\eta}(0, L)$ is imbedded into $H^{*}(0, L)$ for $0 \leq \eta \leq 1 / 2$. Hence,

$$
\int_{\Omega} \psi(X(t, s ; z)) d P \quad \text { is } \mathcal{B}\left(H^{1}(0, L) \times L^{2}(0, L)\right) \text {-measurable in } z .
$$

Next let $\phi$ be a bounded continuous function on $H^{1}(0, L) \times L^{2}(0, L)$. We define $\phi_{m}$ by

$$
\phi_{m}(z)=\phi\left(\left(\Pi_{m} z_{1}, \Pi_{m} z_{2}\right)\right)
$$

where $z=\left(z_{1}, z_{2}\right) \in L^{2}(0, L) \times H^{*}(0, L)$ and $\Pi_{m}$ is the projection onto the subspace spanned by $\left\{e_{1}, \cdots, e_{m}\right\}$. Then, for each $m, \phi_{m}$ is a bounded continuous function on $L^{2}(0, L) \times H^{*}(0, L)$ and for each $y \in \mathcal{Y}$,

$$
\phi_{m}(y) \rightarrow \phi(y) \quad \text { as } m \rightarrow \infty \text {. }
$$

Thus, by (5.33),

$$
\int_{\Omega} \phi_{m}(X(t, s ; z)) d P \quad \text { is } \mathcal{B}\left(H^{1}(0, L) \times L^{2}(0, L)\right) \text {-measurable in } z
$$


and, by (5.34),

$$
\int_{\Omega} \phi(X(t, s ; z)) d P \quad \text { is } \mathcal{B}\left(H^{1}(0, L) \times L^{2}(0, L)\right) \text {-measurable in } z .
$$

Thus, $\mathcal{P}(s, z ; t, \Gamma)$ is $\mathcal{B}\left(H^{1}(0, L) \times L^{2}(0, L)\right)$-measurable in $z$, for each $t>s \geq 0$ and each $\Gamma \in \mathcal{B}\left(H^{1}(0, L) \times L^{2}(0, L)\right)$.

Lemma 5.4. For each $t \geq 0, s \geq 0, z \in \mathcal{Y}$ and $\Gamma \in \mathcal{B}\left(H^{1}(0, L) \times L^{2}(0, L)\right)$, it holds that

$$
\mathcal{P}(0, z ; t, \Gamma)=\mathcal{P}(s, z ; t+s, \Gamma) .
$$

Proof. Fix any $s>0$ and $z=\left(u_{0}, u_{1}\right) \in \mathcal{Y}$. Let us write

$$
B_{j}^{*}(t)=B_{j}(t+s)-B_{j}(s)
$$

and

$$
\Phi^{*}(t)=\Phi(t+s)-\Phi(s)=\sum_{j=1}^{\infty} f_{j} B_{j}^{*}(t) .
$$

Choose any $T>0$, and let $w^{*}$ be the solution of

$$
\left\{\begin{array}{l}
w_{t t}^{*}-w_{x x}^{*}+\alpha w_{t}^{*}=\partial_{t} \Phi^{*} \quad \text { for }(x, t) \in(0, L) \times(0, T), \\
w_{x}^{*}(0, t)=0, \quad w^{*}(L, t)=0 \quad \text { for } t \in(0, T), \\
w^{*}(x, 0)=u_{0}(x), \quad w_{t}^{*}(x, 0)=u_{1}(x) \text { for } x \in(0, L) .
\end{array}\right.
$$

Let $w$ be the solution of (4.12). Then, $w$ and $w^{*}$ have the same distribution in the sense that

$$
P\left(\left(w, w_{t}\right) \in \mathcal{G}\right)=P\left(\left(w^{*}, w_{t}^{*}\right) \in \mathcal{G}\right)
$$

for every $\mathcal{G} \in \mathcal{B}\left(C\left([0, T] ; H^{1}(0, L) \times L^{2}(0, L)\right)\right)$. Let $v_{k}$ and $v_{k}^{*}$ be the solutions in Lemma 2.3 with $K=k$, and $h=w(0, \cdot), h=w^{*}(0, \cdot)$, respectively. The mapping

$$
\left(w, w_{t}\right) \mapsto h \mapsto\left(v_{k}, \partial_{t} v_{k}\right)
$$

is continuous from $C\left([0, T] ; H^{1}(0, L) \times L^{2}(0, L)\right)$ into itself. Therefore, it holds that (5.36)

$$
P\left(\left(w(t)+v_{k}(t), w_{t}(t)+\partial_{t} v_{k}(t)\right) \in \Gamma\right)=P\left(\left(w^{*}(t)+v_{k}^{*}(t), w_{t}^{*}(t)+\partial_{t} v_{k}^{*}(t)\right) \in \Gamma\right)
$$

for every $\Gamma \in \mathcal{B}\left(H^{1}(0, L) \times L^{2}(0, L)\right)$, and every $t \in[0, T]$. Let $\phi$ be a continuous bounded function on $H^{1}(0, L) \times L^{2}(0, L)$. As above, let $\left\{\phi_{n}\right\}_{n=1}^{\infty}$ be a sequence of uniformly bounded functions on $H^{1}(0, L) \times L^{2}(0, L)$ which are continuous with respect to the norm of $L^{2}(0, L) \times H^{*}(0, L)$ such that

$$
\phi_{n}(y) \rightarrow \phi(y) \quad \text { for each } y \in \mathcal{Y} .
$$

It follows from Lemma 5.1 that for almost all $\omega \in \Omega$,

$$
X_{k}=\left(w+v_{k}, \partial_{t} w+\partial_{t} v_{k}\right) \rightarrow X=\left(w+v, w_{t}+v_{t}\right)
$$

weak star in $L^{\infty}\left(0, T ; H^{1}(0, L) \times L^{2}(0, L)\right)$, as $k \rightarrow \infty$, and

$$
X_{k}^{*}=\left(w^{*}+v_{k}^{*}, \partial_{t} w^{*}+\partial_{t} v_{k}^{*}\right) \rightarrow X^{*}=\left(w^{*}+v^{*}, w_{t}^{*}+v_{t}^{*}\right)
$$


weak star in $L^{\infty}\left(0, T ; H^{1}(0, L) \times L^{2}(0, L)\right)$. In the meantime, we can write

$$
\partial_{t}\left(w_{t}+\partial_{t} v_{k}-\Phi\right)=w_{x x}+\partial_{x x} v_{k}-\alpha\left(w_{t}+\partial_{t} v_{k}\right)
$$

and

$$
\partial_{t}\left(w_{t}^{*}+\partial_{t} v_{k}^{*}-\Phi^{*}\right)=w_{x x}^{*}+\partial_{x x} v_{k}^{*}-\alpha\left(w_{t}^{*}+\partial_{t} v_{k}^{*}\right) .
$$

As above, it follows from (5.26) and (5.27) that for almost all $\omega \in \Omega$,

$$
X_{k}=\left(w+v_{k}, \partial_{t} w+\partial_{t} v_{k}\right) \rightarrow X=\left(w+v, w_{t}+v_{t}\right)
$$

strongly in $C\left([0, T] ; L^{2}(0, L) \times H^{*}(0, L)\right)$, as $k \rightarrow \infty$, and

$$
X_{k}^{*}=\left(w^{*}+v_{k}^{*}, \partial_{t} w^{*}+\partial_{t} v_{k}^{*}\right) \rightarrow X^{*}=\left(w^{*}+v^{*}, w_{t}^{*}+v_{t}^{*}\right)
$$

strongly in $C\left([0, T] ; L^{2}(0, L) \times H^{*}(0, L)\right)$. Hence, for each $n \geq 1$, and each $t \in$ $[0, T]$

$$
\int_{\Omega} \phi_{n}\left(X_{k}(t)\right) d P \rightarrow \int_{\Omega} \phi_{n}(X(t)) d P
$$

and

$$
\int_{\Omega} \phi_{n}\left(X_{k}^{*}(t)\right) d P \rightarrow \int_{\Omega} \phi_{n}\left(X^{*}(t)\right) d P,
$$

as $k \rightarrow \infty$. But, by (5.36), we see that for each $n \geq 1$,

$$
\int_{\Omega} \phi_{n}\left(X_{k}(t)\right) d P=\int_{\Omega} \phi_{n}\left(X_{k}^{*}(t)\right) d P .
$$

Thus, for all $n \geq 1$,

$$
\int_{\Omega} \phi_{n}(X(t)) d P=\int_{\Omega} \phi_{n}\left(X^{*}(t)\right) d P .
$$

By passing $n \rightarrow \infty$, we use (5.37) to arrive at

$$
\int_{\Omega} \phi(X(t)) d P=\int_{\Omega} \phi\left(X^{*}(t)\right) d P
$$

which completes the proof of Lemma 5.4.

Next let $\left\{z_{n}\right\}_{n=1}^{\infty}$ be a sequence in $\mathcal{Y}$ such that it is bounded with respect to the norm of $H^{1}(0, L) \times L^{2}(0, L)$, and

$$
z_{n} \rightarrow z \quad \text { in } L^{2}(0, L) \times H^{*}(0, L) .
$$

This implies that $z \in \mathcal{Y}$. Let us write $X_{n}=\left(u_{n}, \partial_{t} u_{n}\right)$ where $u_{n}$ is the solution of $(0.1)-(0.4)$ if the initial condition is $X_{n}(0)=z_{n}$, and write $X=\left(u, u_{t}\right)$ if the initial condition is $X(0)=z$.

Lemma 5.5. For any $T>0$, and any bounded continuous function $\phi$ on $L^{2}(0, L) \times$ $H^{*}(0, L)$,

$$
\int_{\Omega} \phi\left(X_{n}(T)\right) d P \rightarrow \int_{\Omega} \phi(X(T)) d P \quad \text { as } n \rightarrow \infty .
$$

Proof. Fix any $T>0$. Since the sequence $\left\{z_{n}\right\}_{n=1}^{\infty}$ is bounded in $\mathcal{Y}$, it follows from (4.14) - (4.16) and (4.23) that

$$
E\left(\sup _{0 \leq t \leq T}\left\|X_{n}(t)\right\|_{H^{1}(0, L) \times L^{2}(0, L)}^{2}\right)+E\left(\left\|\partial_{x} u_{n}(0, \cdot)\right\|_{L^{2}(0, T)}^{2}\right) \leq M
$$


for all $n \geq 1$, for some positive constant $M$. Choose any $\epsilon>0$. Then there is some constant $K>0$ independent of $n$ such that

$$
P\left\{\sup _{0 \leq t \leq T}\left\|X_{n}(t)\right\|_{H^{1}(0, L) \times L^{2}(0, L)} \geq K\right\}+P\left\{\left\|\partial_{x} u_{n}(0, \cdot)\right\|_{L^{2}(0, T)} \geq K\right\}<\epsilon .
$$

Choose any bounded continuous function $\phi$ on $L^{2}(0, L) \times H^{*}(0, L)$. We assume $\phi \geq 0$, and let

$$
C=\sup _{z \in L^{2}(0, L) \times H^{*}(0, L)} \phi(z),
$$

$$
\mathcal{A}_{n, K}=\left\{\omega \mid \sup _{0 \leq t \leq T}\left\|X_{n}(t)\right\|_{H^{1}(0, L) \times L^{2}(0, L)} \leq K,\left\|\partial_{x} u_{n}(0, \cdot)\right\|_{L^{2}(0, T)} \leq K\right\}
$$

and

$$
\Xi_{n}=\phi\left(X_{n}(T)\right) \chi\left\{\mathcal{A}_{n, K}\right\}
$$

where $\chi\{\cdot\}$ is the characteristic function. We can also define a subset $\Omega^{\dagger} \subset \Omega$ such that $P\left(\Omega \backslash \Omega^{\dagger}\right)=0$ and for each fixed $\omega \in \Omega^{\dagger}$,

$$
\left\{\begin{array}{l}
X(\omega) \text { and } X_{n}(\omega), n \geq 1, \text { belong to } C\left([0, T] ; H^{1}(0, L) \times L^{2}(0, L)\right), \\
\partial_{x} u(0, \cdot) \text { and } \partial_{x} u_{n}(0, \cdot), n \geq 1, \text { belong to } L^{2}(0, T), \\
\Phi(\omega) \in C\left([0, T] ; L^{2}(0, L)\right), \\
u \text { and } u_{n}, n \geq 1, \text { satisfy }(0.1) \text { in the sense of distributions, } \\
u \text { and } u_{n}, n \geq 1, \text { satisfy }(0.2) \text { for almost all } t \in[0, T] .
\end{array}\right.
$$

We will show that for all $\omega \in \Omega^{\dagger}$,

$$
0 \leq \varlimsup_{n \rightarrow \infty} \Xi_{n}(\omega) \leq \phi(X(T, \omega)) .
$$

If $\overline{\lim }_{n \rightarrow \infty} \Xi_{n}(\omega)=0$, then it holds because $\phi \geq 0$. Suppose $\overline{\lim }_{n \rightarrow \infty} \Xi_{n}(\omega)>0$, for some $\omega \in \Omega^{\dagger}$. Then, there is a subsequence $\left\{X_{n_{k}}(\omega)\right\}_{k=1}^{\infty}$ such that

$$
\begin{gathered}
\varlimsup_{n \rightarrow \infty} \Xi_{n}(\omega)=\lim _{k \rightarrow \infty} \Xi_{n_{k}}(\omega)=\lim _{k \rightarrow \infty} \phi\left(X_{n_{k}}(T, \omega)\right), \\
\sup _{0 \leq t \leq T}\left\|X_{n_{k}}(t, \omega)\right\|_{H^{1}(0, L) \times L^{2}(0, L)} \leq K, \\
\left\|\partial_{x} u_{n_{k}}(0, \cdot)\right\|_{L^{2}(0, T)} \leq K, \\
u_{n_{k}} \rightarrow u^{*} \quad \text { weak star in } L^{\infty}\left(0, T ; H^{1}(0, L)\right), \\
\partial_{t} u_{n_{k}} \rightarrow \partial_{t} u^{*} \quad \text { weak star in } L^{\infty}\left(0, T ; L^{2}(0, L)\right),
\end{gathered}
$$

and

$$
\partial_{x} u_{n_{k}}(0, \cdot) \rightarrow \partial_{x} u^{*}(0, \cdot) \quad \text { weakly in } L^{2}(0, T)
$$

where $u^{*}$ satisfies $(0.1)$ in the sense of distributions for this fixed $\omega$. As above, it follows from (0.1), (5.45) and (5.46) that

$$
u_{n_{k}} \rightarrow u^{*} \quad \text { strongly in } C\left([0, T] ; H^{1-\eta}(0, L)\right)
$$

and

$$
\partial_{t} u_{n_{k}} \rightarrow \partial_{t} u^{*} \quad \text { strongly in } C\left([0, T] ; H^{-\eta}(0, L)\right)
$$

for every $\eta>0$. Hence, $u^{*}$ satisfies $(0.2)$ - (0.4). Since $\sigma_{j} \equiv 0, j \geq 1$, we can apply the uniqueness of solution of $(0.1)$ - (0.4) for this fixed $\omega$ to conclude that

$$
X(\omega)=\left(u^{*}, \partial_{t} u^{*}\right)
$$


By (5.48) and (5.49), we see that

$$
X_{n_{k}}(T, \omega) \rightarrow X(T, \omega) \quad \text { in } L^{2}(0, L) \times H^{*}(0, L) .
$$

Hence,

$$
\phi\left(X_{n_{k}}(T, \omega)\right) \rightarrow \phi(X(T, \omega)), \quad \text { as } k \rightarrow \infty
$$

and thus,

$$
\varlimsup_{n \rightarrow \infty} \Xi_{n}(\omega)=\phi(X(T, \omega))
$$

and the inequality holds. Consequently,

$$
\begin{aligned}
& \varlimsup_{n \rightarrow \infty} \int_{\Omega} \Xi_{n}(\omega) d P \leq \int_{\Omega} \varlimsup_{n \rightarrow \infty} \Xi_{n}(\omega) d P \\
& \leq \int_{\Omega} \phi(X(T, \omega)) d P .
\end{aligned}
$$

Hence, by (5.39) and (5.40),

$$
\varlimsup_{n \rightarrow \infty} \int_{\Omega} \phi\left(X_{n}(T, \omega)\right) d P-C \epsilon \leq \int_{\Omega} \phi(X(T, \omega)) d P .
$$

Next let

$$
\Theta_{n}=\phi\left(X_{n}(T)\right) \chi\left\{\mathcal{A}_{n, K}\right\} \vee C\left(1-\chi\left\{\mathcal{A}_{n, K}\right\}\right)
$$

where $C$ is the same constant as in (5.40). We will show that for all $\omega \in \Omega^{\dagger}$,

$$
\phi(X(T, \omega)) \leq \varliminf_{n \rightarrow \infty} \Theta_{n}(\omega) .
$$

If $\underline{\lim }_{n \rightarrow \infty} \Theta_{n}(\omega)=C$, then it is true because of the definition of $C$. Suppose that $\underline{\lim }_{n \rightarrow \infty} \Theta_{n}(\omega)<C$, for some $\omega \in \Omega^{\dagger}$. Then, as above, there is a subsequence $\left\{\Theta_{n_{k}}\right\}_{k=1}^{\infty}$ such that

$$
\varliminf_{n \rightarrow \infty} \Theta_{n}(\omega)=\lim _{k \rightarrow \infty} \Theta_{n_{k}}(\omega)=\lim _{k \rightarrow \infty} \phi\left(X_{n_{k}}(T, \omega)\right)
$$

and (5.43) - (5.49) hold for this $\omega$. By the same argument as above, as $k \rightarrow \infty$,

$$
X_{n_{k}}(T, \omega) \rightarrow X(T, \omega) \quad \text { in } L^{2}(0, L) \times H^{*}(0, L)
$$

and hence,

$$
\varliminf_{n \rightarrow \infty} \Theta_{n}(\omega)=\lim _{k \rightarrow \infty} \phi\left(X_{n_{k}}(T, \omega)\right)=\phi(X(T, \omega)) .
$$

Thus, the inequality holds for all $\omega \in \Omega^{\dagger}$. So it follows from (5.39) and (5.40) that

$$
\begin{aligned}
& \int_{\Omega} \phi(X(T, \omega)) d P \leq \int_{\Omega} \underline{\lim _{n \rightarrow \infty}} \Theta_{n}(\omega) d P \\
& \leq \underset{n \rightarrow \infty}{\lim } \int_{\Omega} \Theta_{n}(\omega) d P \leq \underline{\lim _{n \rightarrow \infty}} \int_{\Omega} \phi\left(X_{n}(T, \omega)\right) d P+C \epsilon .
\end{aligned}
$$

Since $\epsilon>0$ is arbitrary, we have

$$
\lim _{n \rightarrow \infty} \int_{\Omega} \phi\left(X_{n}(T, \omega)\right) d P=\int_{\Omega} \phi(X(T, \omega)) d P .
$$

We can drop the assumption that $\phi \geq 0$, by writing $\phi=\phi^{+}-\phi^{-}$. 
We now check the conditions $[\mathrm{I}]-[\mathrm{V}]$ for the existence of an invariant measure. Here we take $\Xi=H^{1}(0, L) \times L^{2}(0, L)$ and $\Upsilon=L^{2}(0, L) \times H^{*}(0, L) . X(t, s ; z)$ is defined by (5.31) for $t \geq s \geq 0$ and $z \in \mathcal{Y}$. Even though $X(t, s ; z)$ is defined only for $z \in \mathcal{Y}$, this is not a restriction because $X(t, s ; z) \in \mathcal{Y}$ for all $t \geq s \geq 0$, for almost all $\omega$, if $z \in \mathcal{Y}$. Accordingly, the condition on $\left\{\psi_{k}\right\}_{k=1}^{\infty}$ in [IV] is relaxed by

$$
\lim _{k \rightarrow \infty} \psi_{k}(y)=\psi(y), \quad \text { for all } y \in \mathcal{Y},
$$

and the sequence $\left\{z_{n}\right\}_{n=1}^{\infty}$ in [V] is chosen from $\mathcal{S} \cap \mathcal{Y}$. [I] follows from Theorem 1.4 and [II] is verified by Lemmas 5.3 and 5.4. [III] is verified by Lemma 5.2. [V] is verified by Lemma 5.5. It remains to verify [IV]. Let $\psi$ be a bounded continuous function on $\Xi$. We define $\psi_{k}$ by

$$
\psi_{k}(y)=\psi\left(\left(\Pi_{k} y_{1}, \Pi_{k} y_{2}\right)\right), \quad \text { for each } y=\left(y_{1}, y_{2}\right) \in \Upsilon,
$$

where $\Pi_{k}$ is the projection onto the subspace spanned by $\left\{e_{1}, e_{2}, \cdots, e_{k}\right\}$. Then, $\psi_{k}$ satisfies the required property. This completes the proof of Theorem 1.5.

\section{REFERENCES}

[1] Da Prato, G. and Zabczyk, J., Stochastic equations in infinite dimensions, Cambridge University Press, Cambridge, 1992. MR1207136 (95g:60073)

[2] Da Prato, G. and Zabczyk, J., Ergodicity for infinite dimensional systems, Cambridge University Press, Cambridge, 1996. MR1417491 (97k:60165)

[3] Do, C., On the dynamic deformation of a bar against an obstacle, in "Variational methods in the Mechanics of Solids" (1980), pp. $237-241$.

[4] Duvaut, G. and Lions, J.L., Inequalities in Mechanics and Physics, Springer, New YorkBerlin, 1976. MR0521262 (58:25191)

[5] Hausmann, U.G. and Pardoux, E., Stochastic variational inequalities of parabolic type, Applied Math. Opt. 20 (1989), pp. 163 - 193. MR0998402 (90k:60119)

[6] John, F., Partial Differential Equations, 4th edition, Springer-Verlag, New York-HeidelbergBerlin, 1982. MR0831655 (87g:35002)

[7] Kalker, J., Aspects of contact mechanics, in "The Mechanics of the Contact between Deformable Bodies" (1975), pp. 1 - 25.

[8] Karatzas, I. and Shreve, S., Brownian motion and Stochastic Calculus, 2nd edition, Springer, New York-Berlin-Heidelberg, 1997. MR0917065 (89c:60096)

[9] Kim, J.U., A one-dimensional dynamic contact problem in linear viscoelasticity, Math. Methods Appl. Sci. 13 (1990), pp. 55 - 79. MR1060224 (91f:35182)

[10] Kim, J.U., Invariant measures for the stochastic von Karman plate equation, SIAM J. Math. Anal. 36 (2005), pp. 1689 - 1703. MR2139567 (2006c:60073)

[11] Lions, J.L., Quelques méthodes de résolution des problèmes aux limites non linéaires, Dunod, Paris, 1969. MR0259693 (41:4326)

[12] Schatzman, M., A hyperbolic problem of second order with unilateral constraints, J. Math. Anal. Appl. 73 (1980), pp. 138 - 191. MR0560941 (81d:35047)

[13] Schatzman, M., The penalty method for the vibrating string with an obstacle, in "Analytical and Numerical Approaches to Asymptotic Problems in Analysis" (1981), pp. 345 - 357. MR0605520 (82i:35124)

[14] Tulcea, A.I. and Tulcea, C.I., Topics in the Theory of Lifting, Springer-Verlag, New York, 1969. MR0276438 (43:2185)

[15] Villaggio, P., A unilateral contact problem in linear elasticity, J. Elasticity 10 (1980), pp. 113 - 119. MR0576162 (81d:73099)

Department of Mathematics, Virginia Tech, Blacksburg, Virginia 24061-0123

E-mail address: kim@math.vt.edu 